\title{
Psychophysiological Effects of Downregulating Negative Emotions: Insights From a Meta-Analysis of Healthy Adults
}

\author{
Jenny Zaehringer ${ }^{1,2 *}$, Christine Jennen-Steinmetz ${ }^{3}$, Christian Schmahl ${ }^{1,4}$, Gabriele Ende $^{2}$ \\ and Christian Paret ${ }^{1,5 *}$ \\ ${ }^{1}$ Department of Psychosomatic Medicine and Psychotherapy, Central Institute of Mental Health Mannheim, Medical Faculty \\ Mannheim, Heidelberg University, Mannheim, Germany, ${ }^{2}$ Department Neuroimaging, Central Institute of Mental Health \\ Mannheim, Medical Faculty Mannheim, Heidelberg University, Mannheim, Germany, ${ }^{3}$ Department of Biostatistics, Central \\ Institute of Mental Health Mannheim, Medical Faculty Mannheim, Heidelberg University, Mannheim, Germany, ${ }^{4}$ Department \\ of Psychiatry, Schulich School of Medicine and Dentistry, Western University, London, ON, Canada, ${ }^{5}$ Sagol Brain Institute, \\ Wohl Institute for Advanced Imaging, Tel-Aviv Sourasky Medical Centre, Tel-Aviv, Israel
}

\section{OPEN ACCESS}

Edited by:

Carmen Morawetz, Medical University of Vienna, Austria

Reviewed by:

Marcus Grueschow University of Zurich, Switzerland Silvia U. Maier,

University of Zurich, Switzerland

${ }^{*}$ Correspondence:

Jenny Zaehringer jenny.zaehringer@zi-mannheim.de Christian Paret

christian.paret@zi-mannheim.de

Specialty section: This article was submitted to

Emotion Science,

a section of the journal

Frontiers in Psychology

Received: 15 December 2019

Accepted: 28 February 2020

Published: 16 April 2020

Citation:

Zaehringer J, Jennen-Steinmetz C, Schmahl C, Ende G and Paret C (2020) Psychophysiological Effects of Downregulating Negative Emotions: Insights From a Meta-Analysis of Healthy Adults.

Front. Psychol. 11:470. doi: 10.3389/fpsyg.2020.00470
Assessing psychophysiological responses of emotion regulation is a cost-efficient way to quantify emotion regulation and to complement subjective report that may be biased. Previous studies have revealed inconsistent results complicating a sound interpretation of these findings. In the present study, we summarized the existing literature through a systematic search of articles. Meta-analyses were used to evaluate effect sizes of instructed downregulation strategies on common autonomic (electrodermal, respiratory, cardiovascular, and pupillometric) and electromyographic (corrugator activity, emotion-modulated startle) measures. Moderator analyses were conducted, with moderators including study design, emotion induction, control instruction and trial duration. We identified $k=78$ studies each contributing multiple sub-samples and performed 23 meta-analyses for combinations of emotion regulation strategy and psychophysiological measure. Overall, results showed that effects of reappraisal and suppression on autonomic measures were highly inconsistent across studies with rather small mean effect sizes. Electromyography (startle and corrugator activity) showed medium effect sizes that were consistent across studies. Our findings highlight the diversity as well as the low level of standardization and comparability of research in this area. Significant moderation of effects by study design, trial duration, and control condition emphasizes the need for better standardization of methods. In addition, the small mean effect sizes resulting from our analyses on autonomic measures should be interpreted with caution. Findings corroborate the importance of multi-channel approaches.

Keywords: meta-analysis, emotion regulation, psychophysiology, reappraisal, suppression, autonomic nervous system, electromyography

Emotion regulation is a vital part of our daily lives. It permits individuals to control the occurrence, intensity, type, and duration of emotions (Gross and Thompson, 2007). Strategies to regulate emotions not only alter the subjective experience of emotions (Gross, 1998a), but also map onto bodily responses such as changes in measures of the autonomic nervous system (Gross, 2002; Webb et al., 2012), emotion-expressive behavior (Dan-Glauser and Gross, 2011, 2015), somatic reflexes such as the emotion-modulated startle (Jackson et al., 2000), or neural 
activation (Ochsner et al., 2004; Buhle et al., 2014). The habitual use of adaptive emotion regulation strategies is a hallmark of successful functioning and is associated with increased wellbeing, whereas difficulties with regulating emotions have been linked to many psychopathologies (Aldao et al., 2010; Joormann and Vanderlind, 2014; Schmahl et al., 2014). In light of the significance of emotion regulation, appropriate experimental paradigms are required that are suitable for research involving large sample sizes and patient populations.

In a typical emotion regulation study, emotions are experimentally induced using affective stimuli such as films (Gross and Levenson, 1995) or pictures (e.g., International affective picture system; Lang et al., 2009). Participants are instructed to regulate their emotional experience or to respond naturally without regulating their emotions (i.e., the control condition). By comparing the regulation with the control condition it is possible to determine the effect of regulation, which has been used as an indirect measure of emotion regulation effectiveness (Webb et al., 2012).

Assessing psychophysiological correlates has several important advantages. They move beyond on-line self-reports and retrospective assessments, as physiological responding is regarded as automatic, relatively unconscious, and fast (Bradley et al., 1993b; Öhman and Soares, 1994; Edelmann and Baker, 2002; Olsson and Phelps, 2004; Lapate et al., 2014). Research focusing on the direct effects of emotion regulation has found significant psychophysiological changes even when subjective experience remained unaffected (Gross and Levenson, 1993, 1997). Hence, psychophysiological measures can offer important insights into internal emotional experiences that are not available by assessing self-report. In addition, psychophysiological responses are easier to assess than neural physiological measures (e.g., functional magnetic resonance imaging) and are thus cost-efficient methods for quantifying differences in emotion regulation.

\section{CONCEPTUAL FOUNDATIONS OF EMOTION REGULATION}

There have been multiple attempts to classify emotion regulation strategies (Gross, 1998a,b; Larsen, 2000; Koole, 2009). One of the most influential models is the process model of emotion regulation (Gross, 1998a,b, 2015), which broadly categorizes strategies as either being antecedent-focused, i.e., strategies are implemented before the emotional response has fully unfolded, and as response-focused, i.e., strategies are implemented after the emotional response has already been generated. The process model distinguishes five major emotion regulation processes: situation selection (i.e., attempts to change a future emotional response), situation modification (i.e., changing the situation in order to modify its emotional effect), attentional deployment (i.e., distraction away from or concentration on an emotional stimulus to modify the emotion itself), cognitive change (i.e., reappraise a situation or to change the perspective so that the emotional experience is modulated), and response modulation (i.e., strategies to suppress expressive behavior, thoughts, or emotions). Situation selection, situation modification, attentional deployment, and cognitive change are regarded as antecedentfocused and response modulation is regarded as a responsefocused process.

A majority of past emotion regulation studies have instructed participants to distract themselves from, reappraise or suppress ${ }^{1}$ a target stimulus in order to downregulate emotions. These strategies correspond to attentional deployment, cognitive change, and response modulation, respectively, In addition, a considerable number of studies allowed participants to use a strategy of their own choice (Jackson et al., 2000; Dillon and LaBar, 2005; Piper and Curtin, 2006; Lissek et al., 2007; Driscoll et al., 2009; Lee et al., 2009; Golkar et al., 2014; Baur et al., 2015; Conzelmann et al., 2015; Grillon et al., 2015). The present meta-analysis thus focuses on these four major types of downregulation instructions, that is distraction, reappraisal, suppression, and downregulation instructions that allowed participants to choose their own strategy. Other strategies were out of the scope. For a comprehensive overview see Table 1.

\section{PSYCHOPHYSIOLOGICAL RESPONSES OF EMOTIONS AND EMOTION REGULATION}

There is great interest in understanding the relationship between emotions and psychophysiological responses including responses of the autonomic nervous system (i.e., cardiovascular, electrodermal, respiratory, pupillometric) and responses measured with the electromyogram (EMG) such as facial muscle activity (e.g., corrugator supercilii activity) and somatic reflexes (e.g., emotion-modulated startle). The interested reader is directed to detailed reviews by Cacioppo et al. (2000), Kreibig (2010), Siegel et al. (2018), and Stemmler (2004). See Table 2 for an overview of relevant psychophysiological measures within the emotion regulation literature. Such relations have most commonly been studied in terms of two affective dimensions, that is valence (positive-negative) and arousal (high-low) (Lang, 1995; Bradley et al., 2001). Some measures such as heart rate, emotion-modulated startle, and facial activity are specific to the valence of the emotion (Bradley et al., 2001) and others such as skin conductance and pupil dilation are more specific to the arousal dimension (Greenwald et al., 1989; VanOyen Witvliet and Vrana, 1995; Bradley et al., 2001). Past research has also put a lot of effort into answering the question whether different emotion categories (e.g., disgust, sadness, fear) produce distinct physiological response patterns. In a recent metaanalysis the hypothesis could not be confirmed (Siegel et al., 2018). Rather, emotions seem to elicit an unspecific set of psychophysiological changes.

When it comes to the regulation of emotions, much evidence has accumulated suggesting that suppression is related to an

\footnotetext{
${ }^{1}$ Acceptance has become increasingly popular across the emotion regulation literature too, yet there has been a debate as to whether it belongs to antecedent (Webb et al., 2012) or response-focused processes (Hofmann and Asmundson, 2008) and as to whether it is a strategy or rather a function of different strategies. Given that very few studies on acceptance assessed psychophysiological responses, it is not included in the present review.
} 
TABLE 1 | Emotion downregulation processes and their strategies considered in this meta-analysis.

\begin{tabular}{|c|c|c|c|}
\hline Process & Strategy & Subtype & Example \\
\hline \multicolumn{4}{|c|}{ EMOTION REGULATION INSTRUCTIONS } \\
\hline $\begin{array}{l}\text { Attentional } \\
\text { deployment }\end{array}$ & Distraction & Active distraction & $\begin{array}{l}\text { Participants are instructed to think about something positive or neutral that } \\
\text { is unrelated to the target emotion/stimulus }\end{array}$ \\
\hline \multirow[t]{3}{*}{ Cognitive Change } & Reappraisal & Reinterpret the emotional stimulus & $\begin{array}{l}\text { Participants are instructed to reinterpret the emotional stimulus to decrease } \\
\text { the target emotion }\end{array}$ \\
\hline & & $\begin{array}{l}\text { Reappraise via perspective taking, i.e., } \\
\text { distancing }\end{array}$ & $\begin{array}{l}\text { participants are instructed to alter the impact of a stimulus by adopting a } \\
\text { more objective perspective }\end{array}$ \\
\hline & & Reappraise Mixed & A mixture of reappraisal instructions \\
\hline \multirow{4}{*}{$\begin{array}{l}\text { Response } \\
\text { modulation }\end{array}$} & Suppression & Suppress the expression of emotion & Participants are instructed to hide the way they are feeling, e.g., not to smile \\
\hline & & Suppress the experience of an emotion & Participants are instructed to suppress their emotional experience \\
\hline & & $\begin{array}{l}\text { Suppress thoughts of the emotion eliciting } \\
\text { event }\end{array}$ & $\begin{array}{l}\text { Participant are instructed to suppress thoughts about the emotion-eliciting } \\
\text { event }\end{array}$ \\
\hline & & Suppression mixed & A mixture of suppression instructions \\
\hline $\begin{array}{l}\text { Downregulation } \\
\text { unspecified }\end{array}$ & Own choice & Own choice & $\begin{array}{l}\text { Participants are free to choose a strategy that works best for them. They are } \\
\text { not allowed to create a different emotion or think of something unrelated to } \\
\text { the stimulus }\end{array}$ \\
\hline \multicolumn{4}{|c|}{ CONTROL INSTRUCTIONS } \\
\hline & & No instruction (C1) & No instructions are given \\
\hline & & Instructions not to regulate (C2) & Participants are told that they should not use a regulation strategy \\
\hline & & Instructions to maintain (C3) & Participants are instructed to maintain the target emotion \\
\hline & & Instructions to experience naturally (C4) & Participants are instructed to respond naturally without regulating it \\
\hline & & Control mixed (C5) & A mixture of control instructions \\
\hline
\end{tabular}

increase in sympathetic nervous system activity but no difference in self-report to negative stimuli (Gross and Levenson, 1993, 1997; Richards and Gross, 1999). The enhanced sympathetic activation following suppression has led researchers to conclude that suppression "exacts a palpable physiological cost" (Gross and Levenson, 1997, p. 101). In other words, because responsefocused strategies involve an active modulation of expressive behavior, increased sympathetic activation might be the result of that effort (Butler et al., 2003). In contrast, past literature has proposed that reappraisal has little impact on sympathetic and cardiovascular measures (Gross, 1998a). A meta-analysis studying the overall physiological effect of different emotion regulation strategies confirmed this general pattern: cognitive change had a smaller effect on physiology than response modulation (Webb et al., 2012).

However, as noted earlier, there is a vast range of different psychophysiological outcome measures ranging from cardiovascular, electrodermal, respiratory, pupillometric, and electromyographic response systems and it has been shown that the nature of the relationship between cognitive emotion regulation and different psychophysiological responses can vary largely (Bernat et al., 2011). By simply combining all psychophysiological measures to a composite score is helpful in looking at the overall effectiveness of an emotion regulation strategy (as has been done in the meta-analysis by Webb et al., 2012), but it does not reveal which of the individual psychophysiological responses change or do not change with an emotion regulation strategy.

When looking at individual psychophysiological measures, findings are mixed with respect to the effects of emotion regulation on autonomic physiology. Reappraisal instructions focusing on decreasing negative emotions compared to a control condition have been shown to have no effect on (Gross, 1998a; Kalisch et al., 2005; Goldin et al., 2019), increase (Sheppes et al., 2009; Lohani and Isaacowitz, 2014), or decrease (Urry et al., 2009) skin conductance and to increase (Urry et al., 2006; van Reekum et al., 2007) or decrease (Bebko et al., 2011) pupil diameter. Contradictory patterns can also be found for suppression strategies. For example, individuals' heart rate was significantly increased (Hagemann et al., 2006; Ben-Naim et al., 2013), decreased (Gross and Levenson, 1993; Robinson and Demaree, 2009), or stayed the same (Gross, 1998a) when individuals suppressed negative emotions compared to a control condition. These inconsistencies may be due to the large heterogeneity between studies, which can substantially affect the magnitude of the physiological responses. The contradictory pattern of results across the literature does not allow a straightforward interpretation. The causes for these inconsistencies are, however, not wellunderstood, and this inevitably obscures the detection of common trends.

\section{FACTORS RELATED TO THE IMPACT OF EMOTION REGULATION ON PSYCHOPHYSIOLOGY}

\section{Study Design}

Studies using within-study designs found larger effects of emotion regulation on experiential, behavioral and physiological 
TABLE 2 | Common psychophysiological measures of emotion regulation studies.

\begin{tabular}{|c|c|c|c|c|}
\hline $\begin{array}{l}\text { Body } \\
\text { system }\end{array}$ & Measurement & Abbreviation & $\begin{array}{l}\text { Measurement } \\
\text { system (units) }\end{array}$ & Description \\
\hline \multirow[t]{10}{*}{ Cardiovascular } & Cardiac output & $\mathrm{CO}$ & $1 / \min$ & Blood volume pumped by the heart per minute. \\
\hline & Ear pulse transit time & EPTT & ms & $\begin{array}{l}\text { Time interval between the R-wave of the electrocardiogram to the } \\
\text { pulse wave arrival at the ear. }\end{array}$ \\
\hline & Finger pulse transit time & FPTT & ms & $\begin{array}{l}\text { Time interval between the R-wave of the electrocardiogram to the } \\
\text { pulse wave arrival at the finger. }\end{array}$ \\
\hline & $\begin{array}{l}\text { Heart rate/interbeat } \\
\text { interval/heart period }\end{array}$ & HR/HP & $\mathrm{bpm} / \mathrm{ms} / \mathrm{ms}$ & $\begin{array}{l}\text { Number of beats per unit of time/time between heart beats (inverse of } \\
\text { heart rate). }\end{array}$ \\
\hline & Heart rate variability & HRV & $\begin{array}{l}\text { Units vary by } \\
\text { method }\end{array}$ & $\begin{array}{l}\text { Variation in heart rate. Refers specifically to the high-frequency HRV } \\
\text { [also called respiratory sinus arrhythmia (RSA)]. }\end{array}$ \\
\hline & Mean arterial pressure & MAP & $\mathrm{mmHg}$ & $\begin{array}{l}\text { Mean blood pressure of circulating blood on the walls of blood vessels } \\
\text { in between two heartbeats, measured in millimeters of mercury. }\end{array}$ \\
\hline & Pre-ejection period & PEP & $\mathrm{ms}$ & $\begin{array}{l}\text { Period between the beginning of electrical stimulation of the heart to } \\
\text { the opening of the aortic valve. Indicator of the cardiac contractile force } \\
\text { (i.e., how hard the heart is beating). }\end{array}$ \\
\hline & Stroke volume & SV & $\mathrm{mL}$ & Volume of blood pumped from the left ventricle per beat. \\
\hline & Systolic blood pressure & SBP & $\mathrm{mmHg}$ & $\begin{array}{l}\text { Maximum blood pressure of circulating blood on the walls of blood } \\
\text { vessels in between two heartbeats, measured in millimeters of mercury. }\end{array}$ \\
\hline & Total peripheral resistance & TPR & $\begin{array}{l}\text { Unity vary by } \\
\text { method }\end{array}$ & $\begin{array}{l}\text { Overall resistance that must be overcome to push blood through the } \\
\text { whole circulatory system (i.e., all major arterial trees). }\end{array}$ \\
\hline \multirow[t]{4}{*}{ Respiratory } & Inspiration/expiration time & IT/ET & sec & Average inhalation/exhalation time per respiratory cycle. \\
\hline & Respiration amplitude & RA & $\mathbf{m L}$ & $\begin{array}{l}\text { Difference in volts between the point of maximum inspiration and the } \\
\text { point of maximum expiration. }\end{array}$ \\
\hline & Respiration rate & $\mathrm{RR}$ & $\mathrm{c} / \mathrm{min}$ & Number of breaths per minute. \\
\hline & Tidal volume & TV & $\mathrm{mL}$ & Air volume that moves into or out of the lungs while breathing quietly. \\
\hline Pupillometric & Pupil dilation & PD & $\mathbf{m m}$ & Average diameter of pupil in millimeter during a specific period of time. \\
\hline \multirow[t]{3}{*}{ Electromyographic } & $\begin{array}{l}\text { Emotion-modulated } \\
\text { startle }\end{array}$ & Startle & MicroVolt & $\begin{array}{l}\text { Amplitude of the startle eyeblink response (orbicularis oculi) in response } \\
\text { to affective stimuli. }\end{array}$ \\
\hline & $\begin{array}{l}\text { Corrugator supercilii } \\
\text { activity }\end{array}$ & cEMG & MicroVolt & $\begin{array}{l}\text { Muscular activity of the corrugator supercilii responsible for furrowing of } \\
\text { the brow. }\end{array}$ \\
\hline & Zygomaticus major activity & zEMG & MicroVolt & Muscular activity of the zygomaticus major responsible for smiling. \\
\hline Other & Finger temperature & FT & $\mathbf{F} / \mathbf{C}^{\circ}$ & Temperature of the finger, in Fahrenheidt $(\mathrm{F})$ or Celcius $\left(\mathrm{C}^{\circ}\right)$. \\
\hline
\end{tabular}

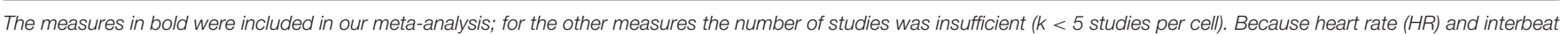

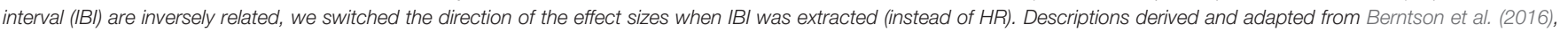
Blumenthal et al. (2005), Cacioppo et al. (2000), Dawson et al. (2016), and Siegel et al. (2018).

outcomes than did studies employing between-study designs (cf. Webb et al., 2012). Employing within-study designs reduces sampling error thereby increasing power. On the other hand, within-study designs may also increase task difficulty because participants are required to engage in more than just one emotion regulation strategy. In event-related designs typical for withinsubject studies, participants may even shift continuously between different strategies. 


\section{Emotion Induction}

Emotion regulation studies have used a variety of different emotional stimuli, including pictures (e.g., the International Affective Picture System; IAPS: Lang et al., 2009), film clips (Gross and Levenson, 1995), stressful tasks (e.g., the Trier Social Stress Test; Kirschbaum et al., 1993), dyadic interactions (Levenson and Gottman, 1983), or threat of shock paradigms (Delgado et al., 2008). Each type of stimulus provides a reliable method to generate emotions. However, a key dimension on which induction methods differ is whether they require participants to sit passively in front of a monitor or whether they employ a stressful task or conversation with a (romantic) partner. Somatic activity has a significant influence on autonomic response measures, especially on heart rate (Obrist, 1981). In addition, stressful tasks such as giving a speech alter the sympathetic nervous system to a stronger degree than picture viewing (Fechir et al., 2008). When it comes to potential differences between films and pictures, findings are mixed. Studies on emotion processing have been shown that e.g., heart rate returns to baseline if the picture remains still, but further slows down if the picture involves motion (Detenber et al., 1998; Simons et al., 1999). However, a recent study on emotion regulation reported that films and pictures did not differently affect the emotion regulation process on a physiological level, although films elicited a stronger absolute skin conductance response than pictures (Morawetz et al., 2016a). We are not aware of any other study directly assessing the impact of the emotion induction method on psychophysiological effects in the context of emotion regulation and thus we will address this question in the present analysis ${ }^{2}$.

\section{Control Instruction}

Effects of emotion regulation strategies on psychophysiological measures can be determined by contrasting the emotion regulation instruction against different control instructions. For example, participants can be instructed to "maintain" the emotion they feel (Jackson et al., 2000), to "view" the emotional stimulus (Gross and Levenson, 1993), or to "respond naturally" (Shiota and Levenson, 2009). Previous literature has shown that differences in neural activation depend on the control condition instruction (Schaefer et al., 2002), with higher amygdala activation reported for "maintain" than for "view" instructions. The terminology used as control instructions (e.g., maintain vs. view) has not been systematically explored in psychophysiological studies of emotion regulation yet. However, it could have important influences on physiological processes as shown by an fMRI study (Diers et al., 2014). Similarly, Webb et al. (2012) found that the control condition moderated the physiological effects of emotion regulation (Webb et al., 2012).

\footnotetext{
${ }^{2}$ It should be noted that there might be more aspects of visual stimuli that could possibly influence effect sizes. For example, within the field of visual perception, studies show that faces are not as evocative as scenes (Alpers et al., 2011; Wangelin et al., 2012). A fine-grained moderator analysis of different aspects of picture and film stimuli however was not possible due to the small number of studies available and because most studies included in the present analysis used a blend of negative scenes and faces as stimuli.
}

\section{Trial Duration}

Another important aspect of the study design which varies largely across studies is the trial duration of the regulation period. According to the implementation and maintenance model (Kalisch, 2009; Paret et al., 2011), reappraisal for example is divided into two phases: In the early phase, participants choose and implement a regulation strategy, whereas in the late phase they maintain the strategy in working memory and monitor its success. Hence, reappraisal might need several seconds until it effectively reduces negative emotions. Thus, the effect of reappraisal might become larger with increasing trial duration, which might also affect physiology.

\section{AIM OF STUDY}

The primary aim of the present study was to quantitatively summarize the relation between popular emotion downregulation instructions (distraction, reappraisal, suppression, own choice) and common psychophysiological measures (i.e., cardiovascular, electrodermal, respiratory, pupillometric, electromyographic) in healthy adults. In light of the contradictory pattern of psychophysiological effects in the emotion regulation literature we aimed to answer the following questions: (a) What are the effects of distraction, reappraisal, suppression, and downregulation where participants choose a strategy that works best for them on individual psychophysiological response measures? (b) How consistent are these effects across studies? and (c) What aspects of the study design moderate the effects? In light of the hypothesis that psychophysiological measures are somewhat sensitive to the valence of the induced emotion and because the majority of studies on emotion regulation and psychophysiology induced negative emotions, the present meta-analysis focuses on the downregulation of negative stimuli (for an overview of studies employing positive stimuli see Table S1).

We first systematically searched for emotion regulation studies that instructed participants to use emotion regulation strategies and that assessed psychophysiological measures of our interest as dependent variable. To advance current knowledge, we performed meta-analyses to separately quantify the effects for each of these measures during emotion regulation. In addition, we performed moderator analyses to explore the impact of study characteristics on the effect sizes. Moderators of interest were study design, trial duration, control instruction, and emotion induction method. It is important to note that our ability to identify the effects of cognitive emotion regulation strategies on psychophysiological variables and potential moderators is limited by the published studies available for meta-analysis.

\section{METHODS}

\section{Selection of Studies}

Studies were identified through a systematic literature search of articles using the PubMed, Web of Science, and PsychINFO databases. The search strategy was developed to maximize the sensitivity of article identification by combining individual words and medical subject headings $(\mathrm{MeSH})^{1}$. We searched for 
the keywords emotion regulation or emotional regulation cross referenced with psychophysiology [MeSH], psychophysiologic*, autonomic, parasympathetic, sympathetic, respiration [MeSH], cardiovascular, electrocardiography $[\mathrm{MeSH}]$, respiratory sinus arrhythmia $[\mathrm{MeSH}]$, blood pressure $[\mathrm{MeSH}]$, heart rate $[\mathrm{MeSH}]$, startle, startle reflex [MeSH], electromyography [MeSH], pupil diameter, pupil dilation, electrodermal or skin conductance, and galvanic skin response $[\mathrm{MeSH}]$ cross referenced with stimulus, stimuli, film* ${ }^{*}$ picture ${ }^{*}$, image $^{*}$, script $^{*}$, anxiety, fear ${ }^{*}$, threat ${ }^{*}$, and video* $^{*}$. Additionally, reference lists from identified studies that met the inclusion criteria (see the next section for criteria) as well as relevant articles in the authors' library were reviewed for titles that might have been previously missed. Subsequently, studies identified in this manner $(n=13)$ were collected for inclusion.

The search process described above yielded a total of 1,353 potentially relevant articles on July 18, 2019 (after duplicates were removed $)^{3}$. The first author and another independent reviewer (Stephanie Mall, research assistant) systematically examined titles and relevant abstracts using the Covidence website (www. covidence.org) to determine whether an article would be subsequently reviewed in full-text format. The following criteria were applied: The study presented original empirical results, was published in a peer-reviewed journal, was written in English or German, included adult healthy participants, and an explicit emotion regulation paradigm was assessed where participants are explicitly told to use emotion regulation strategies to modulate an emotion. We discarded studies that did not assess a psychophysiological measure of interest (e.g., EEG studies) at this point. Based on these criteria, the same two reviewers independently reviewed 157 studies in full-text format.

\section{Inclusion/Exclusion Criteria}

The 157 studies were examined to determine if they met the following inclusion criteria of our analysis: The study (1) included a control condition in which participants were confronted with emotional contents but did not regulate emotions (see Table 1 for definitions of possible control instructions), (2) sampled a psychophysiological measure throughout the regulation phases, (3) did not assess an experimental intervention before the emotion regulation task that may influence the performance of emotion regulation, (4) provided sufficient information to compute the effect size, (5) induced negative emotions, (6) instructed participants to use one or more of the strategies provided in Table 1. If studies met inclusion criteria (1) to (6) but did not provide adequate information for effect size computation, we asked the authors for the needed information via e-mail.

Finally, a total of $n=78$ studies fulfilled all inclusion criteria. Of those, $n=68$ entered our quantitative synthesis (for an overview see Table 3 ). The remaining 10 studies (Delgado et al., 2008; Driscoll et al., 2009; Jamieson et al., 2012, 2013; Peters

\footnotetext{
${ }^{3}$ The search process was updated two times in total. The first search yielded a total of 848 potentially relevant articles on January 22, 2016 (after duplicates were removed). A second search 1 year later (on February 8, 2017) yielded an additional 210 potentially relevant articles (after duplicates were removed). A third search 2 years later (on July 18,2019) yielded an additional 295 potentially relevant articles (after duplicates were removed).
}

et al., 2014; Baur et al., 2015; Reinecke et al., 2015; Peters and Jamieson, 2016; Zaehringer et al., 2018; Kotwas et al., 2019) were not considered, as a meta-analysis on the respective combination of emotion regulation strategy and psychophysiological measure was not possible because the number of studies was too small. See Figure 1 for a PRISMA flowchart depiction of the screening and selection of studies.

\section{Data Extraction}

The first author coded the sample sizes, group means, standard deviations, $t$ and $p$-values for tests on group effects and participants' mean age of the eligible studies. Another person independently coded $50 \%$ of the included studies to evaluate reliability. Correlation analysis confirmed high interraterreliability (mean $r=0.95$, range $=0.66-1.0$ ). In addition, inconsistencies between raters were identified and subsequently corrected. Additionally, the psychophysiological measure, and the specific emotion regulation strategy (distraction, reappraisal, suppression, own choice) were coded. When comparing emotion regulation studies, a major problem arises from inconsistencies in the way emotion regulation instructions are labeled. For example, studies that labeled a condition as "suppression" either instructed participants to use reappraisal (Eippert et al., 2007; Bernat et al., 2011) or to suppress thoughts or facial expressions (Gross and Levenson, 1993; Ohira et al., 2006). To prevent confusion, we specifically evaluated the particular emotion regulation instructions as reported in the articles and coded them according to the taxonomy adapted from Webb et al. (2012). See Table 1 for definitions and examples. For this metaanalysis, we also subdivided the control strategies into five types (classifications can be derived from Table 1; adapted from Webb et al., 2012): no instruction at all (i.e., "view"), instruction "not to regulate in a certain manner," instructions to "respond naturally," instructions to "maintain" the target emotion or a combination of the above instructions. Furthermore, the researcher(s) also coded whether a study used a between-subject design with two independent groups for the control and the experimental group or a within-subject design with a single group undergoing both regulation and control conditions. In addition the nature of emotion induction if applicable [images, film, music, dyadic interaction, past experience or negative self-belief, threat of shock (ToS), stress task, anger task] was also coded. Finally, we coded the trial duration (i.e., the length of the regulation period of a trial, in seconds). We defined the length of a regulation period as the length of one regulation attempt. In event-related designs a regulation attempt thus corresponds to one trial (i.e., after instruction until picture offset), whereas in studies presenting films or stress tasks, a regulation attempt corresponds to the whole film viewing period or task period (i.e., after instruction until end of film/task).

Regarding electrodermal activity, there was great variability in the quantification of skin conductance across studies. We developed a taxonomy by which we divided electrodermal activity measures in skin conductance level, skin conductance response and number of skin conductance responses (see Table 2). A detailed description of the taxonomy and a table summarizing all included studies on electrodermal responses 
TABLE 3 | Characteristics and effect sizes for studies included in the meta-analyses.

\begin{tabular}{|c|c|c|c|c|c|c|c|c|c|c|c|c|}
\hline Study name & Strategy & Measure & Emotion & Design & $\begin{array}{c}\text { Trial } \\
\text { duration (s) }\end{array}$ & $\begin{array}{l}\text { Nature of } \\
\text { emotion } \\
\text { induction }\end{array}$ & $\begin{array}{l}\text { Control } \\
\text { instruction }\end{array}$ & $\mathbf{N}$ total & $\begin{array}{l}\text { Percent of } \\
\text { women }\end{array}$ & $\begin{array}{c}\text { Age } \\
\text { (mean) }\end{array}$ & $\begin{array}{c}\mathrm{N} \\
\text { analyzed }\end{array}$ & $\begin{array}{c}\text { Effect } \\
\text { size }\end{array}$ \\
\hline Ajaya et al. (2016) & Reappraisal & HRV & Anger & B & 120 & Anger task & C1 & 66 & 60.61 & 20.62 & 40 & -0.10 \\
\hline Aldao and Mennin (2012) & Reappraisal & HRV & Disgust, fear, sadness & $\mathrm{B}$ & 62 & $\mathrm{~F}$ & C1 & 58 & 56.90 & 29.57 & 38 & 0.75 \\
\hline Azbel-Jackson et al. (2015), study 1 & Suppression & $\mathrm{HR}$ & Negative & $\mathrm{B}$ & 7 & । & $\mathrm{C} 2$ & 60 & 70.00 & 21.50 & 60 & -0.22 \\
\hline Azbel-Jackson et al. (2015), study 1 & Suppression & $\mathrm{SCL}$ & Negative & $\mathrm{B}$ & 7 & । & $\mathrm{C} 2$ & 60 & 70.00 & 21.50 & 60 & -0.04 \\
\hline Azbel-Jackson et al. (2015), study 2 & Suppression & $\mathrm{HR}$ & Negative & $\mathrm{B}$ & 7 & । & $\mathrm{C} 2$ & 80 & 85.00 & 22.20 & 40 & 0.40 \\
\hline Azbel-Jackson et al. (2015), study 2 & Suppression & $\mathrm{SCL}$ & Negative & $\mathrm{B}$ & 7 & । & $\mathrm{C} 2$ & 80 & 85.00 & 22.20 & 40 & 0.73 \\
\hline Bebko et al. (2011) & Reappraisal & $\mathrm{PD}$ & Negative & W & 10 & । & $\mathrm{C} 4$ & 84 & 47.62 & 19.67 & 40 & -0.09 \\
\hline Ben-Naim et al. (2013) & Reappraisal & FPA & Negative & B & 900 & Dyadic & C1 & 254 & 50.00 & 24.00 & 86 & -1.52 \\
\hline Ben-Naim et al. (2013) & Reappraisal & FPTT & Negative & B & 900 & Dyadic & $\mathrm{C} 1$ & 254 & 50.00 & 24.00 & 86 & -0.18 \\
\hline Ben-Naim et al. (2013) & Reappraisal & $\mathrm{HR}$ & Negative & B & 900 & Dyadic & $\mathrm{C} 1$ & 254 & 50.00 & 24.00 & 86 & 0.33 \\
\hline Ben-Naim et al. (2013) & Reappraisal & $\mathrm{SCL}$ & Negative & B & 900 & Dyadic & $\mathrm{C} 1$ & 254 & 50.00 & 24.00 & 86 & 0.16 \\
\hline Ben-Naim et al. (2013) & Reappraisal & SCR & Negative & B & 900 & Dyadic & $\mathrm{C} 1$ & 254 & 50.00 & 24.00 & 86 & -0.39 \\
\hline Ben-Naim et al. (2013) & Suppression & EPPT & Negative & B & 900 & Dyadic & $\mathrm{C} 1$ & 254 & 50.00 & 24.00 & 85 & 0.09 \\
\hline Ben-Naim et al. (2013) & Suppression & FPA & Negative & B & 900 & Dyadic & C1 & 254 & 50.00 & 24.00 & 85 & -0.66 \\
\hline Ben-Naim et al. (2013) & Suppression & FPTT & Negative & B & 900 & Dyadic & C1 & 254 & 50.00 & 24.00 & 85 & -0.32 \\
\hline Ben-Naim et al. (2013) & Suppression & $\mathrm{HR}$ & Negative & $\mathrm{B}$ & 900 & Dyadic & C1 & 254 & 50.00 & 24.00 & 85 & 0.35 \\
\hline Ben-Naim et al. (2013) & Suppression & $\mathrm{SCL}$ & Negative & $\mathrm{B}$ & 900 & Dyadic & $\mathrm{C} 1$ & 254 & 50.00 & 24.00 & 85 & 0.04 \\
\hline Braams et al. (2012) & Suppression & $\mathrm{HR}$ & Fear & B & 16.5 & ToS & C1 & 123 & 46.34 & 21.70 & 62 & -0.04 \\
\hline Bulut et al. (2018), study 1 & Reappraisal & HRV & Negative & B & 300 & । & $\mathrm{C} 4$ & 28 & 67.86 & 23.67 & 28 & 0.47 \\
\hline Butler et al. (2003), study 1 & Suppression & MAP & Negative & B & & Dyadic & $\mathrm{C} 1$ & 72 & 100.00 & 20.30 & 60 & -0.09 \\
\hline Butler et al. (2006) & Reappraisal & $\mathrm{HR}$ & Negative & B & 590.8 & Dyadic & $\mathrm{C} 1$ & 190 & 100.00 & 20.00 & 62 & -0.24 \\
\hline Butler et al. (2006) & Reappraisal & HRV & Negative & B & 590.8 & Dyadic & $\mathrm{C} 1$ & 190 & 100.00 & 20.00 & 62 & 0.51 \\
\hline Butler et al. (2006) & Reappraisal & RA & Negative & B & 590.8 & Dyadic & $\mathrm{C} 1$ & 190 & 100.00 & 20.00 & 62 & 0.12 \\
\hline Butler et al. (2006) & Suppression & $\mathrm{HR}$ & Negative & $\mathrm{B}$ & 570.6 & Dyadic & $\mathrm{C} 1$ & 190 & 100.00 & 20.00 & 69 & 0.10 \\
\hline Butler et al. (2006) & Suppression & HRV & Negative & $\mathrm{B}$ & 570.6 & Dyadic & $\mathrm{C} 1$ & 190 & 100.00 & 20.00 & 69 & 0.39 \\
\hline Butler et al. (2006) & Suppression & RA & Negative & $\mathrm{B}$ & 570.6 & Dyadic & $\mathrm{C} 1$ & 190 & 100.00 & 20.00 & 69 & -0.76 \\
\hline Butler et al. (2014) & Reappraisal & $\mathrm{SCL}$ & Negative & $\mathrm{B}$ & 590.8 & Dyadic & C1 & 190 & 14.74 & 20.10 & 61 & -0.28 \\
\hline Butler et al. (2014) & Suppression & $\mathrm{SCL}$ & Negative & $\mathrm{B}$ & 570.6 & Dyadic & C1 & 190 & 14.74 & 20.10 & 68 & -0.26 \\
\hline Chu et al. (2019) & Reappraisal & $\mathrm{HR}$ & Anger & $\mathrm{B}$ & 10 & Anger task & C1 & 68 & 54.41 & 40.00 & 68 & -0.14 \\
\hline Colby et al. (1977) & Suppression & $\mathrm{SCL}$ & Fear & W & 6 & ToS & $\mathrm{C} 4$ & 10 & 0.00 & & 10 & -0.11 \\
\hline Conzelmann et al. (2015) & Own choice & Startle & Negative & W & 8 & । & $\mathrm{C} 3$ & 31 & 48.39 & 22.00 & 31 & -0.60 \\
\hline Dan-Glauser and Gross (2011) & Suppression & $\mathrm{FT}$ & Negative & W & 8 & I & $\mathrm{C} 4$ & 37 & 100.00 & 20.20 & 37 & -0.16 \\
\hline Dan-Glauser and Gross (2011) & Suppression & $\mathrm{HR}$ & Negative & W & 8 & । & $\mathrm{C} 4$ & 37 & 100.00 & 20.20 & 37 & -0.57 \\
\hline Dan-Glauser and Gross (2011) & Suppression & MAP & Negative & W & 8 & I & $\mathrm{C} 4$ & 37 & 100.00 & 20.20 & 37 & -0.07 \\
\hline Dan-Glauser and Gross (2011) & Suppression & RA & Negative & W & 8 & । & $\mathrm{C} 4$ & 37 & 100.00 & 20.20 & 37 & -0.82 \\
\hline
\end{tabular}


TABLE 3 | Continued

\begin{tabular}{|c|c|c|c|c|c|c|c|c|c|c|c|c|}
\hline Study name & Strategy & Measure & Emotion & Design & $\begin{array}{c}\text { Trial } \\
\text { duration (s) }\end{array}$ & $\begin{array}{l}\text { Nature of } \\
\text { emotion } \\
\text { induction }\end{array}$ & $\begin{array}{l}\text { Control } \\
\text { instruction }\end{array}$ & $\mathrm{N}$ total & $\begin{array}{c}\text { Percent of } \\
\text { women }\end{array}$ & $\begin{array}{c}\text { Age } \\
\text { (mean) }\end{array}$ & $\begin{array}{c}\mathbf{N} \\
\text { analyzed }\end{array}$ & $\begin{array}{c}\text { Effect } \\
\text { size }\end{array}$ \\
\hline Dan-Glauser and Gross (2015) & Suppression & FPA & Negative & W & 8 & I & $\mathrm{C} 4$ & 37 & 100.00 & 20.20 & 37 & 0.42 \\
\hline Dan-Glauser and Gross (2015) & Suppression & FPTT & Negative & W & 8 & I & $\mathrm{C} 4$ & 37 & 100.00 & 20.20 & 37 & -0.13 \\
\hline Dan-Glauser and Gross (2015) & Suppression & FT & Negative & W & 8 & I & C4 & 37 & 100.00 & 20.20 & 37 & -0.16 \\
\hline Dan-Glauser and Gross (2015) & Suppression & $\mathrm{HR}$ & Negative & W & 8 & I & C4 & 37 & 100.00 & 20.20 & 37 & -0.71 \\
\hline Dan-Glauser and Gross (2015) & Suppression & MAP & Negative & W & 8 & I & C4 & 37 & 100.00 & 20.20 & 37 & -0.50 \\
\hline Dan-Glauser and Gross (2015) & Suppression & RA & Negative & W & 8 & I & $\mathrm{C} 4$ & 37 & 100.00 & 20.20 & 37 & -0.45 \\
\hline Demaree et al. (2006) & Suppression & $\mathrm{HR}$ & Disgust & B & 120 & $\mathrm{~F}$ & C4 & 69 & 52.17 & 19.32 & 35 & 0.09 \\
\hline Demaree et al. (2006) & Suppression & HRV & Disgust & B & 120 & $\mathrm{~F}$ & C4 & 69 & 52.17 & 19.32 & 35 & 0.21 \\
\hline Demaree et al. (2006) & Suppression & RA & Disgust & B & 120 & $\mathrm{~F}$ & C4 & 69 & 52.17 & 19.32 & 35 & 0.43 \\
\hline Demaree et al. (2006) & Suppression & $\mathrm{SCL}$ & Disgust & B & 120 & $\mathrm{~F}$ & C4 & 69 & 52.17 & 19.32 & 35 & 0.12 \\
\hline Denson et al. (2014), study 1 & Reappraisal & $\mathrm{HR}$ & Fear & B & 600 & Stress & $\mathrm{C} 1$ & 90 & 52.22 & 20.54 & 90 & -0.09 \\
\hline Denson et al. (2014), study 1 & Reappraisal & $\mathrm{HR}$ & Fear & B & 300 & Stress & $\mathrm{C} 1$ & 90 & 52.22 & 20.54 & 86 & -0.07 \\
\hline Denson et al. (2011) & Reappraisal & HRV & Anger & B & 180 & $\mathrm{~F}$ & $\mathrm{C} 1$ & 131 & 100.00 & 20.23 & 86 & 0.37 \\
\hline Denson et al. (2011) & Suppression & $\mathrm{HR}$ & Anger & B & 180 & $\mathrm{~F}$ & $\mathrm{C} 1$ & 131 & 100.00 & 20.23 & 89 & 0.25 \\
\hline Denson et al. (2011) & Suppression & HRV & Anger & B & 180 & $\mathrm{~F}$ & $\mathrm{C} 1$ & 131 & 100.00 & 20.23 & 89 & 0.17 \\
\hline Deveney and Pizzagalli (2008) & Reappraisal & cEMG & Negative & W & 5 & I & C3 & 32 & 78.13 & 23.97 & 26 & -0.09 \\
\hline Di Simplicio et al. (2012), sample 1 & Reappraisal & $\mathrm{HR}$ & Negative & W & 4 & I & C4 & 30 & 53.33 & 28.59 & 20 & 0.00 \\
\hline Di Simplicio et al. (2012), sample 1 & Reappraisal & HRV & Negative & W & 4 & I & C4 & 30 & 53.33 & 28.59 & 20 & 0.05 \\
\hline Di Simplicio et al. (2012), sample 2 & Reappraisal & $\mathrm{HR}$ & Negative & W & 4 & I & C4 & 30 & 53.33 & 28.59 & 10 & 0.09 \\
\hline Di Simplicio et al. (2012), sample 2 & Reappraisal & HRV & Negative & W & 4 & 1 & $\mathrm{C} 4$ & 30 & 53.33 & 28.59 & 10 & -0.15 \\
\hline Dillon and LaBar (2005), sample 1 & Own choice & Startle & Negative & W & 12 & I & C3 & 48 & 77.08 & 22.00 & 12 & -0.09 \\
\hline Dillon and LaBar (2005), sample 2 & Own choice & Startle & Negative & W & 12 & I & C3 & 48 & 77.08 & 22.00 & 12 & -0.75 \\
\hline Efinger et al. (2019) & Reappraisal & $\mathrm{HR}$ & Negative & W & 8 & I & C4 & 77 & 100.00 & 20.70 & 77 & -0.27 \\
\hline Efinger et al. (2019) & Reappraisal & RA & Negative & W & 8 & I & C4 & 77 & 100.00 & 20.70 & 77 & 0.06 \\
\hline Efinger et al. (2019) & Reappraisal & $\mathrm{SCL}$ & Negative & W & 8 & I & $\mathrm{C} 4$ & 77 & 100.00 & 20.70 & 77 & -0.19 \\
\hline Efinger et al. (2019) & Distraction & $\mathrm{SCL}$ & Negative & W & 8 & I & $\mathrm{C} 4$ & 77 & 100.00 & 20.70 & 77 & -0.27 \\
\hline Fitzpatrick and Kuo (2016) & Distraction & SCL & Negative & W & 10 & I & & 30 & 66.67 & 30.07 & 30 & 0.00 \\
\hline Fuentes-Sánchez et al. (2019) & Reappraisal & SCR & Negative & W & 8 & I & $\mathrm{C} 4$ & 122 & 59.02 & 25.10 & 106 & -0.01 \\
\hline Goldin et al. (2019) & Reappraisal & $\mathrm{HR}$ & Negative & W & 12 & Self-belief & C4 & 35 & 57.14 & 32.20 & 35 & -0.03 \\
\hline Goldin et al. (2019) & Reappraisal & SCL & Negative & W & 12 & Self-belief & $\mathrm{C} 4$ & 35 & 57.14 & 32.20 & 35 & -0.01 \\
\hline Golkar et al. (2014) & Own choice & Startle & Negative & W & 5 & I & $\mathrm{C} 2$ & 61 & 54.10 & 30.90 & 61 & -0.47 \\
\hline Gomez et al. (2015) & Reappraisal & SCR & Disgust & B & 10 & I & $\mathrm{C} 1$ & 81 & 64.20 & 28.15 & 40 & -0.11 \\
\hline Gross and Levenson (1993), study 1 & Suppression & EPPT & Disgust & B & 64 & $\mathrm{~F}$ & $\mathrm{C} 1$ & 43 & 0.00 & 19.30 & 43 & 0.07 \\
\hline Gross and Levenson (1993), study 1 & Suppression & FPA & Disgust & B & 64 & $\mathrm{~F}$ & $\mathrm{C} 1$ & 43 & 0.00 & 19.30 & 43 & -0.38 \\
\hline Gross and Levenson (1993), study 1 & Suppression & FPTT & Disgust & B & 64 & $\mathrm{~F}$ & $\mathrm{C} 1$ & 43 & 0.00 & 19.30 & 43 & -0.24 \\
\hline Gross and Levenson (1993), study 1 & Suppression & $\mathrm{FT}$ & Disgust & B & 64 & $\mathrm{~F}$ & $\mathrm{C} 1$ & 43 & 0.00 & 19.30 & 43 & -0.30 \\
\hline
\end{tabular}


TABLE 3 | Continued

\begin{tabular}{|c|c|c|c|c|c|c|c|c|c|c|c|c|}
\hline Study name & Strategy & Measure & Emotion & Design & $\begin{array}{c}\text { Trial } \\
\text { duration (s) }\end{array}$ & $\begin{array}{l}\text { Nature of } \\
\text { emotion } \\
\text { induction }\end{array}$ & $\begin{array}{l}\text { Control } \\
\text { instruction }\end{array}$ & $\mathrm{N}$ total & $\begin{array}{l}\text { Percent of } \\
\text { women }\end{array}$ & $\begin{array}{c}\text { Age } \\
\text { (mean) }\end{array}$ & $\begin{array}{c}\mathbf{N} \\
\text { analyzed }\end{array}$ & $\begin{array}{c}\text { Effect } \\
\text { size }\end{array}$ \\
\hline Gross and Levenson (1993), study 1 & Suppression & $\mathrm{HR}$ & Disgust & $\mathrm{B}$ & 64 & $\mathrm{~F}$ & $\mathrm{C} 1$ & 43 & 0.00 & 19.30 & 43 & -0.53 \\
\hline Gross and Levenson (1993), study 1 & Suppression & RA & Disgust & B & 64 & $\mathrm{~F}$ & $\mathrm{C} 1$ & 43 & 0.00 & 19.30 & 43 & -0.18 \\
\hline Gross and Levenson (1993), study 1 & Suppression & SCL & Disgust & B & 64 & $\mathrm{~F}$ & $\mathrm{C} 1$ & 43 & 0.00 & 19.30 & 43 & 0.24 \\
\hline Gross and Levenson (1993), study 2 & Suppression & EPPT & Disgust & B & 64 & $\mathrm{~F}$ & $\mathrm{C} 1$ & 42 & 100.00 & 19.20 & 42 & -0.55 \\
\hline Gross and Levenson (1993), study 2 & Suppression & FPA & Disgust & B & 64 & $\mathrm{~F}$ & $\mathrm{C} 1$ & 42 & 100.00 & 19.20 & 42 & -0.81 \\
\hline Gross and Levenson (1993), study 2 & Suppression & FPTT & Disgust & $\mathrm{B}$ & 64 & $\mathrm{~F}$ & $\mathrm{C} 1$ & 42 & 100.00 & 19.20 & 42 & 0.21 \\
\hline Gross and Levenson (1993), study 2 & Suppression & $\mathrm{FT}$ & Disgust & $\mathrm{B}$ & 64 & $\mathrm{~F}$ & $\mathrm{C} 1$ & 42 & 100.00 & 19.20 & 42 & -0.96 \\
\hline Gross and Levenson (1993), study 2 & Suppression & $\mathrm{HR}$ & Disgust & $\mathrm{B}$ & 64 & $\mathrm{~F}$ & $\mathrm{C} 1$ & 42 & 100.00 & 19.20 & 42 & -0.21 \\
\hline Gross and Levenson (1993), study 2 & Suppression & RA & Disgust & $\mathrm{B}$ & 64 & $\mathrm{~F}$ & $\mathrm{C} 1$ & 42 & 100.00 & 19.20 & 42 & 0.11 \\
\hline Gross and Levenson (1993), study 2 & Suppression & SCL & Disgust & $\mathrm{B}$ & 64 & $\mathrm{~F}$ & $\mathrm{C} 1$ & 42 & 100.00 & 19.20 & 42 & 0.46 \\
\hline Gross and Levenson (1997) & Suppression & $\mathrm{SCL}$ & Sadness & B & 210 & $\mathrm{~F}$ & $\mathrm{C} 1$ & 180 & 100.00 & & 180 & 0.29 \\
\hline Gross (1998a) & Reappraisal & FPA & Disgust & B & 64 & $\mathrm{~F}$ & $\mathrm{C} 1$ & 120 & 50.00 & 21.00 & 80 & 0.12 \\
\hline Gross (1998a) & Reappraisal & $\mathrm{FT}$ & Disgust & B & 64 & $\mathrm{~F}$ & $\mathrm{C} 1$ & 120 & 50.00 & 21.00 & 80 & -0.33 \\
\hline Gross (1998a) & Reappraisal & $\mathrm{HR}$ & Disgust & B & 64 & $\mathrm{~F}$ & $\mathrm{C} 1$ & 120 & 50.00 & 21.00 & 80 & -0.09 \\
\hline Gross (1998a) & Reappraisal & $\mathrm{SCL}$ & Disgust & B & 64 & $\mathrm{~F}$ & $\mathrm{C} 1$ & 120 & 50.00 & 21.00 & 80 & -0.19 \\
\hline Gross (1998a) & Suppression & FPA & Disgust & B & 64 & $\mathrm{~F}$ & $\mathrm{C} 1$ & 120 & 50.00 & 21.00 & 80 & -0.60 \\
\hline Gross (1998a) & Suppression & $\mathrm{FT}$ & Disgust & B & 64 & $\mathrm{~F}$ & $\mathrm{C} 1$ & 120 & 50.00 & 21.00 & 80 & -1.04 \\
\hline Gross (1998a) & Suppression & $\mathrm{HR}$ & Disgust & $\mathrm{B}$ & 64 & $\mathrm{~F}$ & $\mathrm{C} 1$ & 120 & 50.00 & 21.00 & 80 & 0.02 \\
\hline Gross (1998a) & Suppression & $S C L$ & Disgust & B & 64 & $\mathrm{~F}$ & $\mathrm{C} 1$ & 120 & 50.00 & 21.00 & 80 & 0.41 \\
\hline Hagemann et al. (2006) & Suppression & EPPT & Negative & $\mathrm{B}$ & 5 & ToS, I & $\mathrm{C} 1$ & 252 & 51.98 & 20.50 & 168 & -0.38 \\
\hline Hagemann et al. (2006) & Suppression & FPA & Negative & B & 5 & ToS, I & $\mathrm{C} 1$ & 252 & 51.98 & 20.50 & 168 & -0.25 \\
\hline Hagemann et al. (2006) & Suppression & FPTT & Negative & $\mathrm{B}$ & 5 & ToS, I & $\mathrm{C} 1$ & 252 & 51.98 & 20.50 & 168 & -0.39 \\
\hline Hagemann et al. (2006) & Suppression & $\mathrm{FT}$ & Negative & B & 5 & ToS, I & $\mathrm{C} 1$ & 252 & 51.98 & 20.50 & 168 & -0.55 \\
\hline Hagemann et al. (2006) & Suppression & $\mathrm{HR}$ & Negative & $\mathrm{B}$ & 5 & ToS, I & $\mathrm{C} 1$ & 252 & 51.98 & 20.50 & 168 & 0.73 \\
\hline Hagemann et al. (2006) & Suppression & HRV & Negative & $\mathrm{B}$ & 20 & ToS, I & $\mathrm{C} 1$ & 252 & 51.98 & 20.50 & 168 & -0.34 \\
\hline Hagemann et al. (2006) & Suppression & SCL & Negative & B & 5 & ToS, I & $\mathrm{C} 1$ & 252 & 51.98 & 20.50 & 168 & 0.49 \\
\hline Hallam et al. (2015) & Reappraisal & $\mathrm{SCL}$ & Negative & W & 10 & I & $\mathrm{C} 4$ & 40 & 50.00 & 20.00 & 26 & 0.00 \\
\hline Hallam et al. (2015) & Suppression & SCL & Negative & W & 10 & 1 & C4 & 40 & 50.00 & 20.00 & 26 & -0.01 \\
\hline Jackson et al. (2000) & Own choice & Startle & Negative & W & 14 & I & C3 & 48 & 68.75 & 20.50 & 44 & -1.04 \\
\hline Kim and Hamann (2012) & Reappraisal & cEMG & Negative & W & 24 & I & $\mathrm{C} 4$ & 36 & 50.00 & 20.19 & 33 & -0.30 \\
\hline Kim and Hamann (2012) & Reappraisal & SCR & Negative & W & 24 & 1 & C4 & 36 & 50.00 & 20.19 & 32 & 0.11 \\
\hline Kinner et al. (2017) & Reappraisal & PD & Negative & W & 5 & I & $\mathrm{C} 4$ & 30 & 100.00 & 24.40 & 28 & 0.26 \\
\hline Kinner et al. (2017) & Reappraisal & SCR & Negative & W & 5 & I & $\mathrm{C} 4$ & 30 & 100.00 & 24.40 & 25 & 0.00 \\
\hline Kunzmann et al. (2005) & Suppression & $\mathrm{HR}$ & Disgust & W & 117 & $\mathrm{~F}$ & $\mathrm{C} 1$ & 95 & 49.47 & 46.00 & 47 & -0.26 \\
\hline Kunzmann et al. (2005) & Suppression & $\mathrm{SCL}$ & Disgust & W & 117 & $\mathrm{~F}$ & $\mathrm{C} 1$ & 95 & 49.47 & 46.00 & 47 & 0.15 \\
\hline Leiberg et al. (2012) & Reappraisal & SCR & Negative & W & 6 & 1 & C4 & 24 & 100.00 & 24.10 & 24 & 0.17 \\
\hline
\end{tabular}




\begin{tabular}{|c|c|c|c|c|c|c|c|c|c|c|c|c|}
\hline Study name & Strategy & Measure & Emotion & Design & $\begin{array}{c}\text { Trial } \\
\text { duration (s) }\end{array}$ & $\begin{array}{l}\text { Nature of } \\
\text { emotion } \\
\text { induction }\end{array}$ & $\begin{array}{l}\text { Control } \\
\text { instruction }\end{array}$ & $\mathrm{N}$ total & $\begin{array}{c}\text { Percent of } \\
\text { women }\end{array}$ & $\begin{array}{c}\text { Age } \\
\text { (mean) }\end{array}$ & $\begin{array}{c}\mathrm{N} \\
\text { analyzed }\end{array}$ & $\begin{array}{c}\text { Effect } \\
\text { size }\end{array}$ \\
\hline Lohani and Isaacowitz (2014), sample 1 & Reappraisal & CEMG & Sadness & W & 300 & $\mathrm{~F}$ & C1 & 48 & 79.17 & 71.42 & 42 & -0.17 \\
\hline Lohani and Isaacowitz (2014), sample 1 & Reappraisal & $\mathrm{SCL}$ & Sadness & W & 300 & $\mathrm{~F}$ & $\mathrm{C} 1$ & 42 & 73.81 & 18.50 & 40 & 0.56 \\
\hline Lohani and Isaacowitz (2014), sample 1 & Suppression & $\mathrm{SCL}$ & Sadness & W & 300 & $\mathrm{~F}$ & C1 & 42 & 73.81 & 18.50 & 40 & 0.52 \\
\hline Lohani and Isaacowitz (2014), sample 2 & Reappraisal & cEMG & Sadness & W & 300 & $\mathrm{~F}$ & C1 & 42 & 73.81 & 18.50 & 40 & -0.30 \\
\hline Lohani and Isaacowitz (2014), sample 2 & Reappraisal & $\mathrm{SCL}$ & Sadness & W & 300 & $\mathrm{~F}$ & $\mathrm{C} 1$ & 48 & 79.17 & 71.42 & 44 & 0.09 \\
\hline Lohani and Isaacowitz (2014), sample 2 & Suppression & $\mathrm{SCL}$ & Sadness & W & 300 & $\mathrm{~F}$ & $\mathrm{C} 1$ & 48 & 79.17 & 71.42 & 44 & 0.13 \\
\hline Lohani and Isaacowitz (2014), sample 1 & Distraction & $\mathrm{SCL}$ & Sadness & W & 300 & $\mathrm{~F}$ & $\mathrm{C} 1$ & 42 & 73.81 & 18.50 & 40 & 0.48 \\
\hline Lohani and Isaacowitz (2014), sample 2 & Distraction & SCL & Sadness & W & 300 & $\mathrm{~F}$ & C1 & 48 & 79.17 & 71.42 & 44 & 0.24 \\
\hline Low et al. (2008) & Reappraisal & $\mathrm{HR}$ & Negative & B & 600 & Stress & C3 & 81 & 58.02 & 20.60 & 56 & 0.29 \\
\hline Martins et al. (2018) & Reappraisal & PD & Negative & W & 7 & 1 & C4 & 48 & 68.75 & 69.10 & 48 & 0.06 \\
\hline Martins et al. (2018) & Reappraisal & PD & Negative & W & 7 & 1 & C4 & 48 & 60.42 & 21.06 & 48 & 0.06 \\
\hline Morawetz et al. (2016a) & Reappraisal & SCR & Negative & W & 8 & $\mathrm{I}, \mathrm{F}$ & C4 & 59 & 33.90 & 32.47 & 47 & 0.08 \\
\hline Morawetz et al. (2016b) & Reappraisal & SCR & Negative & W & 8 & 1 & C4 & 23 & 52.17 & 25.70 & 16 & -0.19 \\
\hline Morawetz et al. (2017) & Reappraisal & SCR & Negative & W & 8 & $\mathrm{~F}$ & C4 & 23 & 65.22 & 22.95 & 22 & -0.03 \\
\hline Ohira et al. (2006) & Suppression & $\mathrm{HR}$ & Negative & W & 60 & 1 & C4 & 10 & 100.00 & 24.22 & 9 & 0.04 \\
\hline Opitz et al. (2014), sample 1 & Reappraisal & CEMG & Sadness & W & 8 & 1 & $\mathrm{C} 4$ & 30 & 53.33 & 61.90 & 29 & -0.43 \\
\hline Opitz et al. (2014), sample 1 & Reappraisal & $\mathrm{HR}$ & Sadness & W & 8 & 1 & $\mathrm{C} 4$ & 30 & 63.33 & 19.45 & 28 & -0.02 \\
\hline Opitz et al. (2014), sample 1 & Reappraisal & $\mathrm{SCL}$ & Sadness & W & 8 & 1 & $\mathrm{C} 4$ & 30 & 63.33 & 19.45 & 27 & -0.02 \\
\hline Opitz et al. (2014), sample 2 & Reappraisal & cEMG & Sadness & W & 8 & 1 & $\mathrm{C} 4$ & 30 & 63.33 & 19.45 & 28 & -1.07 \\
\hline Opitz et al. (2014), sample 2 & Reappraisal & $\mathrm{HR}$ & Sadness & W & 8 & 1 & $\mathrm{C} 4$ & 30 & 53.33 & 61.90 & 29 & -0.14 \\
\hline Opitz et al. (2014), sample 2 & Reappraisal & SCL & Sadness & W & 8 & 1 & C4 & 30 & 53.33 & 61.90 & 29 & -0.27 \\
\hline Ortner (2015) & Reappraisal & SCR & Negative & $\mathrm{B}$ & 8 & 1 & $C 1$ & 120 & 75.83 & & 76 & 0.01 \\
\hline Plieger et al. (2017) & Reappraisal & $\mathrm{SCL}$ & Negative & W & 4.5 & 1 & C1 & 91 & 82.42 & 24.53 & 91 & -0.28 \\
\hline Richards and Gross (1999), study2 & Suppression & DBP & Negative & B & 84 & 1 & $\mathrm{C} 1$ & 85 & 100.00 & 18.80 & 74 & 0.36 \\
\hline Richards and Gross (1999), study2 & Suppression & FT & Negative & $\mathrm{B}$ & 84 & 1 & C1 & 85 & 100.00 & 18.80 & 74 & -0.37 \\
\hline Richards and Gross (1999), study2 & Suppression & $\mathrm{HR}$ & Negative & $\mathrm{B}$ & 84 & 1 & $\mathrm{C} 1$ & 85 & 100.00 & 18.80 & 74 & -0.11 \\
\hline Richards and Gross (1999), study2 & Suppression & SBP & Negative & B & 84 & 1 & C1 & 85 & 100.00 & 18.80 & 74 & 0.27 \\
\hline Richards and Gross (1999), study2 & Suppression & SCL & Negative & $\mathrm{B}$ & 84 & I & C1 & 85 & 100.00 & 18.80 & 74 & -0.14 \\
\hline Roberts et al. (2008), sample 1 & Suppression & DBP & Disgust & $\mathrm{B}$ & 62 & $\mathrm{~F}$ & C1 & 40 & 60.00 & 20.80 & 40 & 0.91 \\
\hline Roberts et al. (2008), sample 1 & Suppression & $\mathrm{HR}$ & Disgust & $\mathrm{B}$ & 62 & $\mathrm{~F}$ & C1 & 40 & 60.00 & 20.80 & 40 & -0.23 \\
\hline Roberts et al. (2008), sample 1 & Suppression & SBP & Disgust & B & 62 & $\mathrm{~F}$ & C1 & 40 & 60.00 & 20.80 & 40 & 0.60 \\
\hline Roberts et al. (2008), sample 1 & Suppression & $\mathrm{SCL}$ & Negative & $\mathrm{B}$ & 62 & $\mathrm{~F}$ & C1 & 40 & 60.00 & 20.80 & 40 & 0.00 \\
\hline Roberts et al. (2008), sample 2 & Suppression & DBP & Disgust & B & 62 & $\mathrm{~F}$ & C1 & 40 & 60.00 & 20.80 & 40 & 0.84 \\
\hline Roberts et al. (2008), sample 2 & Suppression & $\mathrm{HR}$ & Disgust & B & 62 & $\mathrm{~F}$ & C1 & 40 & 60.00 & 20.80 & 40 & 0.08 \\
\hline Roberts et al. (2008), sample 2 & Suppression & SBP & Disgust & $\mathrm{B}$ & 62 & $\mathrm{~F}$ & $C 1$ & 40 & 60.00 & 20.80 & 40 & 0.66 \\
\hline Roberts et al. (2008), sample 2 & Suppression & $\mathrm{SCL}$ & Negative & B & 62 & $\mathrm{~F}$ & C1 & 40 & 60.00 & 20.80 & 40 & 0.35 \\
\hline Roberts et al. (2008), sample 3 & Suppression & DBP & Disgust & B & 62 & $\mathrm{~F}$ & C1 & 40 & 60.00 & 20.80 & 40 & -0.31 \\
\hline
\end{tabular}




\begin{tabular}{|c|c|c|c|c|c|c|c|c|c|c|c|c|}
\hline Study name & Strategy & Measure & Emotion & Design & $\begin{array}{c}\text { Trial } \\
\text { duration (s) }\end{array}$ & $\begin{array}{l}\text { Nature of } \\
\text { emotion } \\
\text { induction }\end{array}$ & $\begin{array}{l}\text { Control } \\
\text { instruction }\end{array}$ & $\mathbf{N}$ total & $\begin{array}{l}\text { Percent of } \\
\text { women }\end{array}$ & $\begin{array}{c}\text { Age } \\
\text { (mean) }\end{array}$ & $\begin{array}{c}\mathrm{N} \\
\text { analyzed }\end{array}$ & $\begin{array}{l}\text { Effect } \\
\text { size }\end{array}$ \\
\hline Roberts et al. (2008), sample 3 & Suppression & $\mathrm{HR}$ & Disgust & $\mathrm{B}$ & 62 & $\mathrm{~F}$ & C1 & 40 & 60.00 & 20.80 & 40 & -0.61 \\
\hline Roberts et al. (2008), sample 3 & Suppression & SBP & Disgust & $\mathrm{B}$ & 62 & $\mathrm{~F}$ & C1 & 40 & 60.00 & 20.80 & 40 & 0.01 \\
\hline Roberts et al. (2008), sample 3 & Suppression & SCL & Negative & B & 62 & $\mathrm{~F}$ & C1 & 40 & 60.00 & 20.80 & 40 & 0.62 \\
\hline Roberts et al. (2008), sample 4 & Suppression & DBP & Disgust & $\mathrm{B}$ & 62 & $\mathrm{~F}$ & C1 & 40 & 60.00 & 20.80 & 40 & 0.12 \\
\hline Roberts et al. (2008), sample 4 & Suppression & $\mathrm{HR}$ & Disgust & B & 62 & $\mathrm{~F}$ & C1 & 40 & 60.00 & 20.80 & 40 & 0.26 \\
\hline Roberts et al. (2008), sample 4 & Suppression & SBP & Disgust & B & 62 & $\mathrm{~F}$ & C1 & 40 & 60.00 & 20.80 & 40 & 0.11 \\
\hline Roberts et al. (2008), sample 4 & Suppression & SCL & Negative & $\mathrm{B}$ & 62 & $\mathrm{~F}$ & C1 & 40 & 60.00 & 20.80 & 40 & 0.30 \\
\hline Robinson and Demaree (2009) & Suppression & $\mathrm{HR}$ & Sadness & W & 120 & $\mathrm{~F}$ & C4 & 102 & 50.98 & 19.75 & 102 & -0.23 \\
\hline Robinson and Demaree (2009) & Suppression & HRV & Sadness & W & 120 & $\mathrm{~F}$ & C4 & 102 & 50.98 & 19.75 & 102 & 0.41 \\
\hline Robinson and Demaree (2009) & Suppression & SCL & Sadness & W & 120 & $\mathrm{~F}$ & C4 & 102 & 50.98 & 19.75 & 102 & 0.26 \\
\hline Rohrmann et al. (2009), sample 1 & Reappraisal & $\mathrm{HR}$ & Disgust & B & 60 & $\mathrm{~F}$ & C1 & 120 & 0.00 & 25.47 & 36 & 0.22 \\
\hline Rohrmann et al. (2009), sample 2 & Reappraisal & $\mathrm{HR}$ & Disgust & $\mathrm{B}$ & 60 & $\mathrm{~F}$ & C1 & 120 & 0.00 & 25.47 & 36 & -0.34 \\
\hline Rohrmann et al. (2009), sample 1 & Suppression & $\mathrm{HR}$ & Disgust & $\mathrm{B}$ & 60 & $\mathrm{~F}$ & C1 & 120 & 0.00 & 25.47 & 36 & 0.47 \\
\hline Rohrmann et al. (2009), sample 2 & Suppression & $\mathrm{HR}$ & Disgust & $\mathrm{B}$ & 60 & $\mathrm{~F}$ & C1 & 120 & 0.00 & 25.47 & 36 & -0.66 \\
\hline Rohrmann et al. (2009), sample 1 & Reappraisal & SCL & Disgust & $\mathrm{B}$ & 60 & $\mathrm{~F}$ & C1 & 120 & 0.00 & 25.47 & 36 & 0.35 \\
\hline Rohrmann et al. (2009), sample 2 & Reappraisal & SCL & Disgust & $\mathrm{B}$ & 60 & $\mathrm{~F}$ & $\mathrm{C} 1$ & 120 & 0.00 & 25.47 & 36 & -0.57 \\
\hline Rohrmann et al. (2009), sample 1 & Suppression & SCL & Disgust & $\mathrm{B}$ & 60 & $\mathrm{~F}$ & C1 & 120 & 0.00 & 25.47 & 36 & 0.85 \\
\hline Rohrmann et al. (2009), sample 2 & Suppression & SCL & Disgust & $\mathrm{B}$ & 60 & $\mathrm{~F}$ & C1 & 120 & 0.00 & 25.47 & 36 & -0.23 \\
\hline Roth et al. (2014), study2 & Suppression & SCL & Fear & $\mathrm{B}$ & 197 & $\mathrm{~F}$ & C1 & 116 & 60.34 & 24.90 & 65 & -0.04 \\
\hline Roth et al. (2014), study2 & Distraction & $\mathrm{SCL}$ & Fear & $\mathrm{B}$ & 197 & $\mathrm{~F}$ & $\mathrm{C} 1$ & 116 & 60.34 & 24.90 & 67 & -0.77 \\
\hline Sheppes et al. (2009) & Reappraisal & $\mathrm{FT}$ & Sadness & B & 190 & $\mathrm{~F}$ & C5 & 45 & 100.00 & 22.90 & 29 & 0.22 \\
\hline Sheppes et al. (2009) & Reappraisal & SCL & Sadness & $\mathrm{B}$ & 190 & $\mathrm{~F}$ & C5 & 45 & 100.00 & 22.90 & 29 & 1.13 \\
\hline Sheppes et al. (2009) & Distraction & SCL & Sadness & $\mathrm{B}$ & 190 & $\mathrm{~F}$ & C5 & 45 & 100.00 & 22.90 & 29 & 0.23 \\
\hline Shermohammed et al. (2017) & Reappraisal & $H R$ & Negative & W & 8 & I & C1 & 25 & 48.00 & 20.89 & 19 & 0.65 \\
\hline Shermohammed et al. (2017) & Reappraisal & SCR & Negative & W & 8 & । & C1 & 25 & 48.00 & 20.89 & 17 & 0.12 \\
\hline Shiota and Levenson (2009, 2012), sample 1 & Suppression & DBP & Disgust & W & 180 & $\mathrm{~F}$ & C4 & 76 & 50.00 & 25.50 & 73 & -0.66 \\
\hline Shiota and Levenson (2009, 2012), sample 1 & Suppression & EPPT & Disgust & W & 180 & $\mathrm{~F}$ & C4 & 76 & 50.00 & 25.50 & 74 & 0.33 \\
\hline Shiota and Levenson (2009, 2012), sample 1 & Suppression & FPA & Disgust & w & 180 & $\mathrm{~F}$ & C4 & 76 & 50.00 & 25.50 & 75 & 0.49 \\
\hline Shiota and Levenson (2009, 2012), sample 1 & Suppression & FPTT & Disgust & w & 180 & $\mathrm{~F}$ & $\mathrm{C} 4$ & 76 & 50.00 & 25.50 & 75 & -0.12 \\
\hline Shiota and Levenson (2009, 2012), sample 1 & Suppression & FT & Disgust & W & 180 & $\mathrm{~F}$ & C4 & 76 & 50.00 & 25.50 & 76 & -0.24 \\
\hline Shiota and Levenson (2009, 2012), sample 1 & Suppression & $\mathrm{HR}$ & Disgust & w & 180 & $\mathrm{~F}$ & $\mathrm{C} 4$ & 76 & 50.00 & 25.50 & 75 & -0.40 \\
\hline Shiota and Levenson (2009, 2012), sample 1 & Suppression & MAP & Disgust & W & 180 & $\mathrm{~F}$ & C4 & 76 & 50.00 & 25.50 & 73 & -0.66 \\
\hline Shiota and Levenson (2009, 2012), sample 1 & Suppression & RA & Disgust & W & 180 & $\mathrm{~F}$ & C4 & 76 & 50.00 & 25.50 & 72 & -0.29 \\
\hline Shiota and Levenson (2009, 2012), sample 1 & Suppression & SBP & Disgust & W & 180 & $\mathrm{~F}$ & C4 & 76 & 50.00 & 25.50 & 73 & -0.69 \\
\hline Shiota and Levenson (2009, 2012), sample 1 & Suppression & SCL & Disgust & W & 180 & $\mathrm{~F}$ & C4 & 76 & 50.00 & 25.50 & 73 & -0.42 \\
\hline Shiota and Levenson (2009, 2012), sample 2 & Reappraisal & FPA & Disgust, sadness & W & 180 & $\mathrm{~F}$ & C4 & 22 & 50.00 & 25.50 & 23 & 0.37 \\
\hline Shiota and Levenson $(2009,2012)$, sample 2 & Reappraisal & FPTT & Disgust, sadness & W & 180 & $\mathrm{~F}$ & C4 & 22 & 50.00 & 25.50 & 23 & 0.47 \\
\hline
\end{tabular}




\begin{tabular}{|c|c|c|c|c|c|c|c|c|c|c|c|c|}
\hline Study name & Strategy & Measure & Emotion & Design & $\begin{array}{c}\text { Trial } \\
\text { duration (s) }\end{array}$ & $\begin{array}{l}\text { Nature of } \\
\text { emotion } \\
\text { induction }\end{array}$ & $\begin{array}{l}\text { Control } \\
\text { instruction }\end{array}$ & $\mathrm{N}$ total & $\begin{array}{l}\text { Percent of } \\
\text { women }\end{array}$ & $\begin{array}{c}\text { Age } \\
\text { (mean) }\end{array}$ & $\begin{array}{c}\mathrm{N} \\
\text { analyzed }\end{array}$ & $\begin{array}{l}\text { Effect } \\
\text { size }\end{array}$ \\
\hline Shiota and Levenson $(2009,2012)$, sample 2 & Reappraisal & FT & Disgust, sadness & W & 180 & $\mathrm{~F}$ & $\mathrm{C} 4$ & 22 & 50.00 & 25.50 & 23 & 0.36 \\
\hline Shiota and Levenson $(2009,2012)$, sample 2 & Reappraisal & $\mathrm{HR}$ & Disgust, sadness & W & 180 & $\mathrm{~F}$ & $\mathrm{C} 4$ & 22 & 50.00 & 25.50 & 23 & -0.29 \\
\hline Shiota and Levenson (2009, 2012), sample 2 & Reappraisal & RA & Disgust, sadness & W & 180 & $\mathrm{~F}$ & $\mathrm{C} 4$ & 22 & 50.00 & 25.50 & 22 & -0.34 \\
\hline Shiota and Levenson (2009, 2012), sample 2 & Reappraisal & SCL & Disgust, sadness & W & 180 & $\mathrm{~F}$ & C4 & 22 & 50.00 & 25.50 & 23 & -0.27 \\
\hline Shiota and Levenson $(2009,2012)$, sample 3 & Reappraisal & FPA & Disgust, sadness & W & 180 & $\mathrm{~F}$ & $\mathrm{C} 4$ & 26 & 50.00 & 25.30 & 25 & 0.14 \\
\hline Shiota and Levenson $(2009,2012)$, sample 3 & Reappraisal & FPTT & Disgust, sadness & W & 180 & $\mathrm{~F}$ & $\mathrm{C} 4$ & 26 & 50.00 & 25.30 & 25 & 0.02 \\
\hline Shiota and Levenson (2009, 2012), sample 3 & Reappraisal & FT & Disgust, sadness & W & 180 & $\mathrm{~F}$ & $\mathrm{C} 4$ & 26 & 50.00 & 25.30 & 26 & 0.12 \\
\hline Shiota and Levenson (2009, 2012), sample 3 & Reappraisal & $\mathrm{HR}$ & Disgust, sadness & W & 180 & $\mathrm{~F}$ & $\mathrm{C} 4$ & 26 & 50.00 & 25.30 & 25 & -0.11 \\
\hline Shiota and Levenson (2009, 2012), sample 3 & Reappraisal & RA & Disgust, sadness & W & 180 & $\mathrm{~F}$ & $\mathrm{C} 4$ & 26 & 50.00 & 25.30 & 24 & -0.10 \\
\hline Shiota and Levenson (2009, 2012), sample 3 & Reappraisal & SCL & Disgust, sadness & W & 180 & $\mathrm{~F}$ & $\mathrm{C} 4$ & 26 & 50.00 & 25.30 & 24 & 0.10 \\
\hline Shiota and Levenson (2009, 2012), sample 4 & Suppression & DBP & Disgust & w & 180 & $\mathrm{~F}$ & $\mathrm{C} 4$ & 72 & 50.00 & 44.70 & 64 & -0.27 \\
\hline Shiota and Levenson (2009, 2012), sample 4 & Suppression & EPPT & Disgust & W & 180 & $\mathrm{~F}$ & $\mathrm{C} 4$ & 72 & 50.00 & 44.70 & 71 & -0.06 \\
\hline Shiota and Levenson (2009, 2012), sample 4 & Suppression & FPA & Disgust & W & 180 & $\mathrm{~F}$ & $\mathrm{C} 4$ & 72 & 50.00 & 44.70 & 71 & 0.27 \\
\hline Shiota and Levenson (2009, 2012), sample 4 & Suppression & FPTT & Disgust & W & 180 & $\mathrm{~F}$ & $\mathrm{C} 4$ & 72 & 50.00 & 44.70 & 72 & 0.11 \\
\hline Shiota and Levenson (2009, 2012), sample 4 & Suppression & FT & Disgust & W & 180 & $\mathrm{~F}$ & $\mathrm{C} 4$ & 72 & 50.00 & 44.70 & 72 & -0.03 \\
\hline Shiota and Levenson (2009, 2012), sample 4 & Suppression & $\mathrm{HR}$ & Disgust & w & 180 & $\mathrm{~F}$ & $\mathrm{C} 4$ & 72 & 50.00 & 44.70 & 72 & -0.30 \\
\hline Shiota and Levenson (2009, 2012), sample 4 & Suppression & MAP & Disgust & W & 180 & $\mathrm{~F}$ & $\mathrm{C} 4$ & 72 & 50.00 & 44.70 & 64 & -0.28 \\
\hline Shiota and Levenson (2009, 2012), sample 4 & Suppression & RA & Disgust & w & 180 & $\mathrm{~F}$ & $\mathrm{C} 4$ & 72 & 50.00 & 44.70 & 66 & -0.07 \\
\hline Shiota and Levenson (2009, 2012), sample 4 & Suppression & SBP & Disgust & W & 180 & $\mathrm{~F}$ & $\mathrm{C} 4$ & 72 & 50.00 & 44.70 & 64 & -0.32 \\
\hline Shiota and Levenson (2009, 2012), sample 4 & Suppression & SCL & Disgust & w & 180 & $\mathrm{~F}$ & $\mathrm{C} 4$ & 72 & 50.00 & 44.70 & 69 & -0.39 \\
\hline Shiota and Levenson (2009, 2012), sample 5 & Reappraisal & FPA & Disgust, sadness & W & 180 & $\mathrm{~F}$ & $\mathrm{C} 4$ & 22 & 50.00 & 44.70 & 23 & 0.23 \\
\hline Shiota and Levenson (2009, 2012), sample 5 & Reappraisal & FPTT & Disgust, sadness & W & 180 & $\mathrm{~F}$ & $\mathrm{C} 4$ & 22 & 50.00 & 44.70 & 24 & -0.28 \\
\hline Shiota and Levenson (2009, 2012), sample 5 & Reappraisal & FT & Disgust, sadness & W & 180 & $\mathrm{~F}$ & $\mathrm{C} 4$ & 22 & 50.00 & 44.70 & 24 & 0.00 \\
\hline Shiota and Levenson (2009, 2012), sample 5 & Reappraisal & $H R$ & Disgust, sadness & W & 180 & $\mathrm{~F}$ & C4 & 22 & 50.00 & 44.70 & 24 & -0.31 \\
\hline Shiota and Levenson (2009, 2012), sample 5 & Reappraisal & RA & Disgust, sadness & W & 180 & $\mathrm{~F}$ & $\mathrm{C} 4$ & 22 & 50.00 & 44.70 & 23 & -0.18 \\
\hline Shiota and Levenson (2009, 2012), sample 5 & Reappraisal & SCL & Disgust, sadness & W & 180 & $\mathrm{~F}$ & $\mathrm{C} 4$ & 22 & 50.00 & 44.70 & 22 & -0.10 \\
\hline Shiota and Levenson (2009, 2012), sample 6 & Reappraisal & FPA & Disgust, sadness & W & 180 & $\mathrm{~F}$ & $\mathrm{C} 4$ & 26 & 50.00 & 43.20 & 26 & 0.17 \\
\hline Shiota and Levenson (2009, 2012), sample 6 & Reappraisal & FPT & Disgust, sadness & w & 180 & $\mathrm{~F}$ & $\mathrm{C} 4$ & 26 & 50.00 & 43.20 & 26 & 0.21 \\
\hline Shiota and Levenson (2009, 2012), sample 6 & Reappraisal & FT & Disgust, sadness & w & 180 & $\mathrm{~F}$ & $\mathrm{C} 4$ & 26 & 50.00 & 43.20 & 26 & 0.23 \\
\hline Shiota and Levenson (2009, 2012), sample 6 & Reappraisal & $\mathrm{HR}$ & Disgust, sadness & w & 180 & $\mathrm{~F}$ & $\mathrm{C} 4$ & 26 & 50.00 & 43.20 & 26 & -0.06 \\
\hline Shiota and Levenson (2009, 2012), sample 6 & Reappraisal & RA & Disgust, sadness & w & 180 & $\mathrm{~F}$ & $\mathrm{C} 4$ & 26 & 50.00 & 43.20 & 24 & -0.10 \\
\hline Shiota and Levenson (2009, 2012), sample 6 & Reappraisal & $\mathrm{SCL}$ & Disgust, sadness & W & 180 & $\mathrm{~F}$ & $\mathrm{C} 4$ & 26 & 50.00 & 43.20 & 25 & -0.09 \\
\hline Shiota and Levenson (2009, 2012), sample 7 & Suppression & DBP & Disgust & W & 180 & $\mathrm{~F}$ & $\mathrm{C} 4$ & 72 & 50.00 & 64.80 & 69 & -0.30 \\
\hline Shiota and Levenson (2009, 2012), sample 7 & Suppression & EPPT & Disgust & W & 180 & $\mathrm{~F}$ & $\mathrm{C} 4$ & 72 & 50.00 & 64.80 & 68 & -0.01 \\
\hline Shiota and Levenson (2009, 2012), sample 7 & Suppression & FPA & Disgust & W & 180 & $\mathrm{~F}$ & $\mathrm{C} 4$ & 72 & 50.00 & 64.80 & 65 & 0.23 \\
\hline Shiota and Levenson (2009, 2012), sample 7 & Suppression & FPTT & Disgust & W & 180 & $\mathrm{~F}$ & $\mathrm{C} 4$ & 72 & 50.00 & 64.80 & 65 & 0.16 \\
\hline Shiota and Levenson $(2009,2012)$, sample 7 & Suppression & FT & Disgust & W & 180 & $\mathrm{~F}$ & $\mathrm{C} 4$ & 72 & 50.00 & 64.80 & 72 & 0.11 \\
\hline
\end{tabular}




\begin{tabular}{|c|c|c|c|c|c|c|c|c|c|c|c|c|}
\hline Study name & Strategy & Measure & Emotion & Design & $\begin{array}{c}\text { Trial } \\
\text { duration (s) }\end{array}$ & $\begin{array}{l}\text { Nature of } \\
\text { emotion } \\
\text { induction }\end{array}$ & $\begin{array}{l}\text { Control } \\
\text { instruction }\end{array}$ & $\mathrm{N}$ total & $\begin{array}{c}\text { Percent of } \\
\text { women }\end{array}$ & $\begin{array}{c}\text { Age } \\
\text { (mean) }\end{array}$ & $\begin{array}{c}\mathbf{N} \\
\text { analyzed }\end{array}$ & $\begin{array}{c}\text { Effect } \\
\text { size }\end{array}$ \\
\hline Shiota and Levenson $(2009,2012)$, sample 7 & Suppression & $\mathrm{HR}$ & Disgust & W & 180 & $\mathrm{~F}$ & C4 & 72 & 50.00 & 64.80 & 69 & -0.12 \\
\hline Shiota and Levenson $(2009,2012)$, sample 7 & Suppression & MAP & Disgust & W & 180 & $\mathrm{~F}$ & C4 & 72 & 50.00 & 64.80 & 69 & -0.30 \\
\hline Shiota and Levenson $(2009,2012)$, sample 7 & Suppression & RA & Disgust & W & 180 & $\mathrm{~F}$ & C4 & 72 & 50.00 & 64.80 & 66 & -0.26 \\
\hline Shiota and Levenson $(2009,2012)$, sample 7 & Suppression & SBP & Disgust & W & 180 & $\mathrm{~F}$ & C4 & 72 & 50.00 & 64.80 & 69 & -0.27 \\
\hline Shiota and Levenson $(2009,2012)$, sample 7 & Suppression & $\mathrm{SCL}$ & Disgust & W & 180 & $\mathrm{~F}$ & $\mathrm{C} 4$ & 72 & 50.00 & 64.80 & 69 & -0.46 \\
\hline Shiota and Levenson $(2009,2012)$, sample 8 & Reappraisal & FPA & Disgust, sadness & W & 180 & $\mathrm{~F}$ & C4 & 24 & 50.00 & 64.80 & 23 & -0.08 \\
\hline Shiota and Levenson $(2009,2012)$, sample 8 & Reappraisal & FPTT & Disgust, sadness & W & 180 & $\mathrm{~F}$ & C4 & 24 & 50.00 & 64.80 & 23 & 0.03 \\
\hline Shiota and Levenson $(2009,2012)$, sample 8 & Reappraisal & FT & Disgust, sadness & W & 180 & $\mathrm{~F}$ & C4 & 24 & 50.00 & 64.80 & 24 & 0.10 \\
\hline Shiota and Levenson $(2009,2012)$, sample 8 & Reappraisal & $\mathrm{HR}$ & Disgust, sadness & W & 180 & $\mathrm{~F}$ & C4 & 24 & 50.00 & 64.80 & 23 & -0.19 \\
\hline Shiota and Levenson $(2009,2012)$, sample 8 & Reappraisal & RA & Disgust, sadness & W & 180 & $\mathrm{~F}$ & $\mathrm{C} 4$ & 24 & 50.00 & 64.80 & 20 & -0.19 \\
\hline Shiota and Levenson $(2009,2012)$, sample 8 & Reappraisal & $\mathrm{SCL}$ & Disgust, sadness & W & 180 & $\mathrm{~F}$ & C4 & 24 & 50.00 & 64.80 & 23 & -0.11 \\
\hline Shiota and Levenson $(2009,2012)$, sample 9 & Reappraisal & FPA & Disgust, sadness & W & 180 & $\mathrm{~F}$ & $\mathrm{C} 4$ & 24 & 50.00 & 64.50 & 22 & 0.40 \\
\hline Shiota and Levenson $(2009,2012)$, sample 9 & Reappraisal & FPTT & Disgust, sadness & W & 180 & $\mathrm{~F}$ & $\mathrm{C} 4$ & 24 & 50.00 & 64.50 & 22 & -0.12 \\
\hline Shiota and Levenson $(2009,2012)$, sample 9 & Reappraisal & $\mathrm{FT}$ & Disgust, sadness & W & 180 & $\mathrm{~F}$ & $\mathrm{C} 4$ & 24 & 50.00 & 64.50 & 23 & 0.58 \\
\hline Shiota and Levenson $(2009,2012)$, sample 9 & Reappraisal & $\mathrm{HR}$ & Disgust, sadness & W & 180 & $\mathrm{~F}$ & C4 & 24 & 50.00 & 64.50 & 22 & -0.10 \\
\hline Shiota and Levenson $(2009,2012)$, sample 9 & Reappraisal & RA & Disgust, sadness & W & 180 & $\mathrm{~F}$ & C4 & 24 & 50.00 & 64.50 & 22 & -0.26 \\
\hline Shiota and Levenson $(2009,2012)$, sample 9 & Reappraisal & SCL & Disgust, sadness & W & 180 & $\mathrm{~F}$ & C4 & 24 & 50.00 & 64.50 & 22 & -0.64 \\
\hline Soto et al. (2016) & Suppression & $\mathrm{HR}$ & Disgust & W & 58 & $\mathrm{~F}$ & $\mathrm{C} 1$ & 59 & 54.24 & 19.51 & 48 & -0.19 \\
\hline Soto et al. (2016) & Suppression & SCL & Disgust & W & 58 & $\mathrm{~F}$ & $\mathrm{C} 1$ & 59 & 54.24 & 19.51 & 47 & -0.15 \\
\hline Stiller et al. (2019) & Reappraisal & $\mathrm{HR}$ & Negative & $\mathrm{B}$ & 165 & $\mathrm{~F}$ & C2 & 61 & 73.77 & 24.30 & 41 & 0.15 \\
\hline Stiller et al. (2019) & Reappraisal & $\mathrm{SCL}$ & Negative & B & 165 & $\mathrm{~F}$ & $\mathrm{C} 2$ & 61 & 73.77 & 24.30 & 41 & 0.49 \\
\hline Stiller et al. (2019) & Suppression & $\mathrm{HR}$ & Negative & B & 165 & $\mathrm{~F}$ & $\mathrm{C} 2$ & 61 & 73.77 & 24.30 & 40 & 0.58 \\
\hline Stiller et al. (2019) & Suppression & $\mathrm{SCL}$ & Negative & B & 165 & $\mathrm{~F}$ & $\mathrm{C} 2$ & 61 & 73.77 & 24.30 & 40 & 0.35 \\
\hline Strauss et al. (2016) & Reappraisal & PD & Negative & W & 5 & 1 & $\mathrm{C} 4$ & 25 & 64.00 & 19.80 & 25 & 0.14 \\
\hline Svaldi et al. (2010) & Reappraisal & FPTT & Sadness & W & 125 & $\mathrm{~F}$ & C1 & 25 & 100.00 & 38.30 & 21 & -0.11 \\
\hline Svaldi et al. (2010) & reappraisal & $\mathrm{HR}$ & Sadness & W & 125 & $\mathrm{~F}$ & $\mathrm{C} 1$ & 25 & 100.00 & 38.30 & 25 & -0.32 \\
\hline Svaldi et al. (2010) & reappraisal & $\mathrm{HRV}$ & Sadness & W & 125 & $\mathrm{~F}$ & C1 & 25 & 100.00 & 38.30 & 21 & -0.67 \\
\hline Svaldi et al. (2010) & reappraisal & SCL & Sadness & W & 125 & $\mathrm{~F}$ & C1 & 25 & 100.00 & 38.30 & 23 & 0.10 \\
\hline Svaldi et al. (2010) & Suppression & FPTT & Sadness & W & 211 & $\mathrm{~F}$ & C1 & 25 & 100.00 & 38.30 & 21 & -0.68 \\
\hline Svaldi et al. (2010) & Suppression & $\mathrm{HR}$ & Sadness & W & 211 & $\mathrm{~F}$ & C1 & 25 & 100.00 & 38.30 & 25 & -0.16 \\
\hline Svaldi et al. (2010) & Suppression & HRV & Sadness & W & 211 & $\mathrm{~F}$ & C1 & 25 & 100.00 & 38.30 & 21 & -0.18 \\
\hline Svaldi et al. (2010) & Suppression & $\mathrm{SCL}$ & Sadness & W & 211 & $\mathrm{~F}$ & $\mathrm{C} 1$ & 25 & 100.00 & 38.30 & 23 & 0.50 \\
\hline Urry et al. (2006) & Reappraisal & PD & Negative & W & 5 & 1 & C3 & 17 & 52.94 & 62.90 & 14 & 0.43 \\
\hline Urry et al. (2009) & Reappraisal & PD & Negative & W & 8 & 1 & C3 & 26 & 57.69 & 64.80 & 26 & 0.46 \\
\hline Urry et al. (2009) & Reappraisal & SCL & Negative & W & 8 & 1 & $\mathrm{C} 3$ & 26 & 57.69 & 64.80 & 26 & -0.42 \\
\hline Urry (2009) & Reappraisal & cEMG & Negative & W & 8 & 1 & $\mathrm{C} 2$ & 41 & 63.41 & 20.00 & 40 & 0.03 \\
\hline Urry (2009) & Reappraisal & $\mathrm{HR}$ & Negative & W & 8 & 1 & C2 & 41 & 63.41 & 20.00 & 40 & -0.14 \\
\hline
\end{tabular}




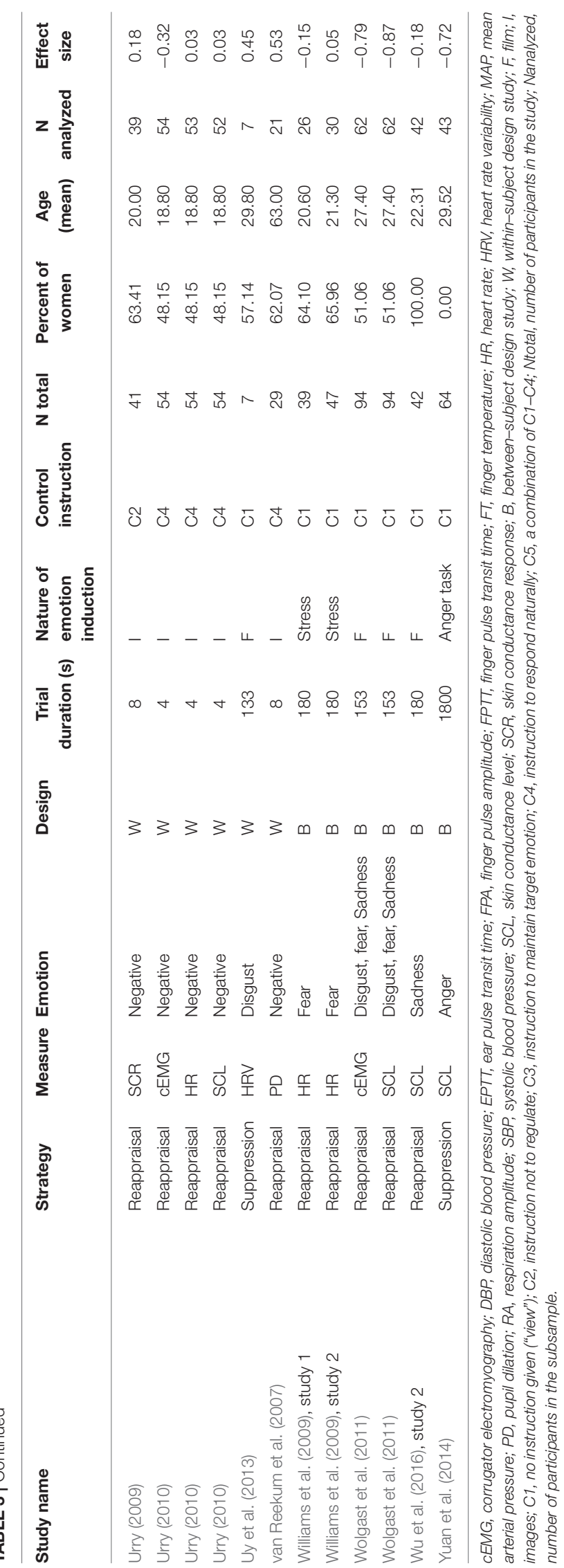

with information about the categorization can be found in the supplement (p. 2 and Table S2).

\section{Statistical Analysis}

Cohen's $d$ was used as the effect size measure in the metaanalyses. For between-subject studies, effect sizes were calculated from the means and standard deviations of the control and experimental (regulation) groups. For within-subject studies, we used the means and standard deviations of the control and experimental (regulation) conditions. If these values were not available, effect sizes were calculated using $t$-values. Furthermore, the variances of the effect sizes were determined. In withinsubject designs, the variance of the effect size estimate depends on the correlation between the paired measurements. If the correlation was not available from the original data, the median correlation from the other studies entering the meta-analysis was used. Effect sizes were interpreted based on Cohen's guidelines (Cohen, 1988). Therefore, effects at the 0.2, 0.5, and 0.8 levels were considered as small, medium, and large, respectively.

Since the experimental conditions of the studies differ in many ways, it is unlikely that the studies share a common effect size. Fixed-effect models are therefore implausible. Following recommendations of Borenstein et al. (2010) we conducted random effects meta-analyses. We calculated average effect sizes and $95 \%$ confidence intervals (CI). Heterogeneity of effect sizes was assessed with the $I^{2}$-statistic which represents the proportion of total variation in the estimated effect sizes that is due to heterogeneity between studies (Higgins and Thompson, 2002). The analyses were performed separated by psychophysiological measure and emotion regulation strategy. Meta-analyses were only conducted when five or more independent samples were available ${ }^{4}$.

For each significant meta-analysis we constructed a funnel plot with the effect sizes on the horizontal axis and their standard errors on the vertical axis. Egger's tests (Egger et al., 1997) were applied to evaluate asymmetry in funnel plots which may be caused by publication bias.

Several studies included two or three assessments within a given measure (e.g., skin conductance level during the regulation of sad and disgusting stimuli) so that there was more than one effect size reported for a specific sample. In these cases, we used the mean of the multiple effect sizes. To calculate the variance of this mean effect size, we assumed that the correlation between the effect sizes was 0.5. If studies reported sufficient results from multiple independent samples (e.g., men and women, prone to disgust vs. not prone to disgust), each of them entered the analysis. Effect sizes for interbeat interval and heart rate were included in the same analyses. To align to polarity of the effect sizes, the parameter for interbeat interval was multiplied by minus one. Thus, a negative size of interbeat interval corresponds to decreased heart rate.

As physiological measures have been shown to discriminate between negative and positive emotional states (Levenson et al., 1990; Bradley and Lang, 2000; Kreibig, 2010), we aimed for

${ }^{4}$ Some studies included several independent samples. The minimum number of independent studies required to conduct a meta-analysis was accepted as three. 


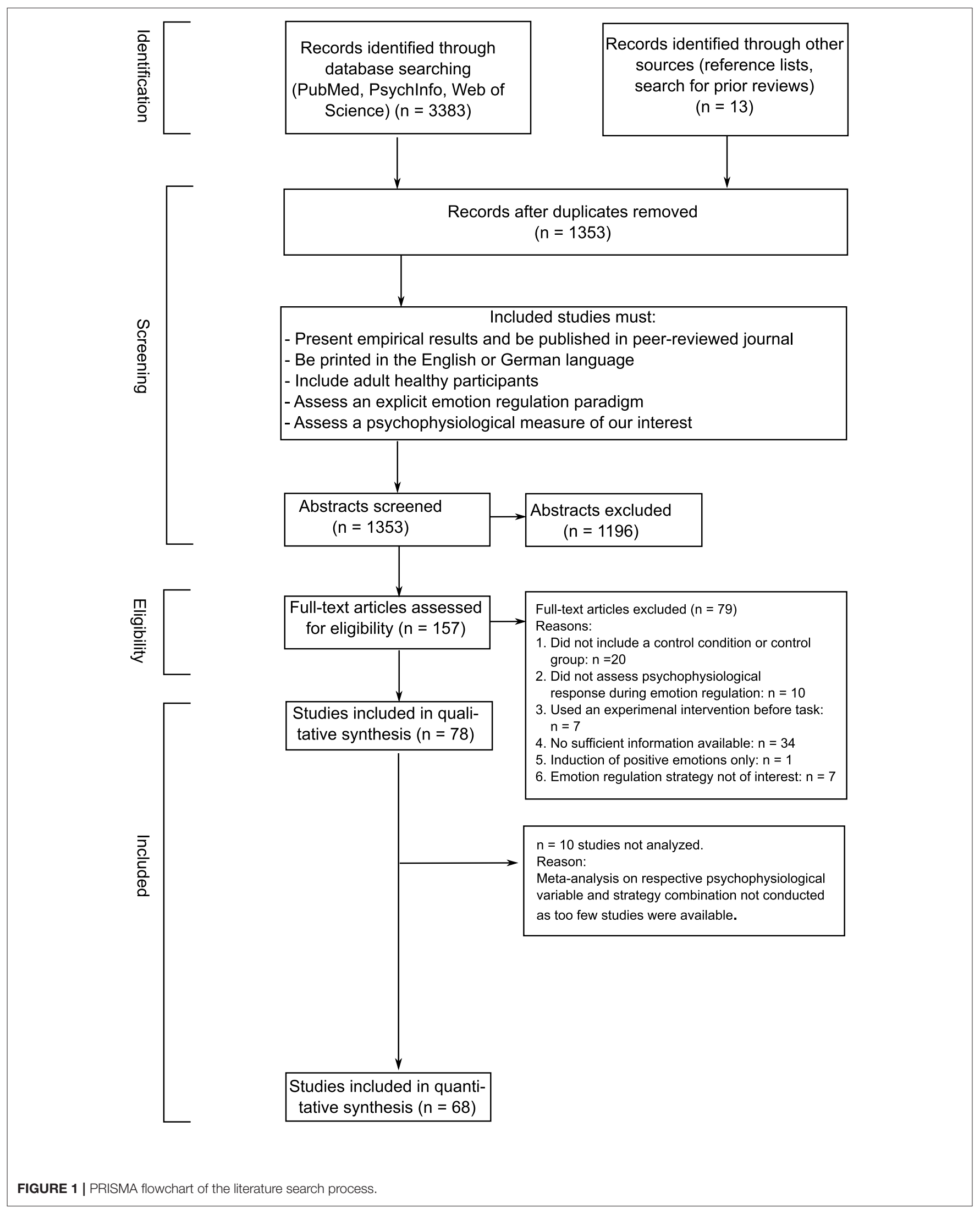


distinguishing between positive and negative target emotions in our analyses. Only 13 studies in total (Gross and Levenson, 1997; Demaree et al., 2004; Ohira et al., 2006; Giuliani et al., 2008; Driscoll et al., 2009; Dan-Glauser and Gross, 2011, 2015; Gruber et al., 2014; Baur et al., 2015; Conzelmann et al., 2015; Gomez et al., 2015; Wu et al., 2016; Kotwas et al., 2019) induced positive emotions. Combinations of psychophysiological measure and emotion regulation strategy resulted in a maximum of three studies. Therefore, meta-analyses on the regulation of positive emotions were not computed in the present study. See an overview of studies using positive emotions in the Table S1.

We conducted moderator analyses to test whether features of the experimental context influenced the effect sizes. We used four moderator variables in our analyses: study design (within-subject vs. between-subject), nature of control condition (instruction to respond naturally vs. no instruction), nature of emotion induction (films vs. pictures), and trial duration (i.e., length of a regulation trial, in seconds), as far as there were enough studies for statistical comparison. To evaluate the effects of moderators we used meta-regression analyses and present the regression coefficients.

Statistical analyses were conducted with the metaphor package from R (version 3.2) and SAS 9.4 (SAS Institute Inc., Cary, NC, USA). Statistical significance was defined at the $5 \%$ level.

\section{Heterogeneity}

We investigated whether the variance between the observed effect sizes was larger than what would be expected on the basis of sampling variance alone (Hedges, 1982; Rosenthal and Rubin, 1982). If the effect sizes are heterogeneous it means that the mean effect size does not represent individual effect sizes for studies within the population in that moderators of the effect sizes may be present (e.g., nature of emotion induction). In an analysis with a small number of effect sizes, especially if they are based on small sample size studies, the Q-statistic may be nonsignificant even when there is considerable variability among the effect sizes. Therefore, we computed the percent of variability in effect sizes due to heterogeneity using the $I^{2}$ statistic (Higgins and Thompson, 2002). I represents the amount of variability in effect sizes that is accounted for by heterogeneity as a proportion of the total variability. According to Higgins and Thompson's (2002) general guidelines, mild heterogeneity would be suggested by an $I^{2}=30 \%$ of the variability in effect sizes, moderate heterogeneity by an $I^{2}$ between 30 and $50 \%$, and notable heterogeneity when $I^{2}$ is $>50 \%$ of the variability.

\section{Moderator Analyses}

We conducted moderator analyses to test whether features of the experimental context influenced the observed effect sizes. We used four moderator variables in our analyses: study design (within-subject vs. between-subject), nature of control condition (instruction to respond naturally vs. no instruction) ${ }^{5}$,

${ }^{5}$ We were unable to test other types of control instructions as there were too few studies available. nature of emotion induction (films vs. pictures) ${ }^{6}$, trial duration (i.e., length of a regulation trial, in seconds), as far as there were sufficient cases for statistical comparison. We used metaregression (Thompson and Sharp, 1999) to evaluate moderators. The advantage of meta-regression is that continuous moderators (e.g., trial duration) can be evaluated alongside categorical moderators (e.g., within- vs. between-participants designs). For the meta- regressions, $\beta$ is the beta weight or coefficient assigned to the predictor; $t$ (and the associated $p$-value) tests whether the beta weight is significantly different from zero.

\section{RESULTS}

\section{Descriptive Analyses}

Across the 78 studies that were initially considered in our qualitative analysis, heart rate (HR) and skin conductance level (SCL) was measured most frequently, with three times as many effect sizes as for any other measure (see Figure 2 for an overview). Thus, emotion regulation strategies and psychophysiological measures were not evenly represented in the published literature. Certain combinations of emotion regulation strategy and psychophysiological measures occurred frequently in published experiments (e.g., reappraisal and measuring heart rate) whereas other combinations were rare or non-existent (e.g., suppression while measuring stroke volume).

Sixty-nine individual studies entered our quantitative analyses (for a flowchart of the selection and screening process see Figure 1). Study characteristics of these studies are presented in Table 3. There are $n=4,474$ unique individuals across all of the 68 included studies (meaning that this is the total $n$ across all studies) with many individuals contributing data to more than one effect size for a total of $n=13,380$ data points across all meta-analytic comparisons. Because not all studies reported demographic statistics, reported information about age and sex is only an estimated number.

\section{Meta-Analyses}

As the 68 studies contributed data to multiple effect sizes, we computed 267 individual effect sizes (see Table 3) that entered 24 different meta-analyses (see Table 4 and Figure 3). Overall, computed individual mean effect sizes for each combination of regulation strategy with measure did not exceed $\mathrm{d}=$ 0.62 (own choice effect on startle; see Table 4). Figure 3 also highlights that some meta-analyses revealed large confidence intervals and non-significant effect sizes, suggesting that these effects are rather inconsistent (e.g., suppression effect on skin conductance response, ear pulse transit time, diastolic blood pressure and finger pulse amplitude, reappraisal effect on finger pulse amplitude, heart rate variability, and distraction effect on skin conductance level). Largest effect sizes were obtained for electromyographic responses (startle and corrugator activity), followed by suppression effects on some cardiovascular measures (i.e., finger temperature and mean arterial pressure). For many

\footnotetext{
${ }^{6}$ We were unable to test other types of emotion inductions (i.e., music, dyadic interaction, past experience or personally relevant thought, threat of shock, stressor task, anger task) as there were too few studies available.
} 


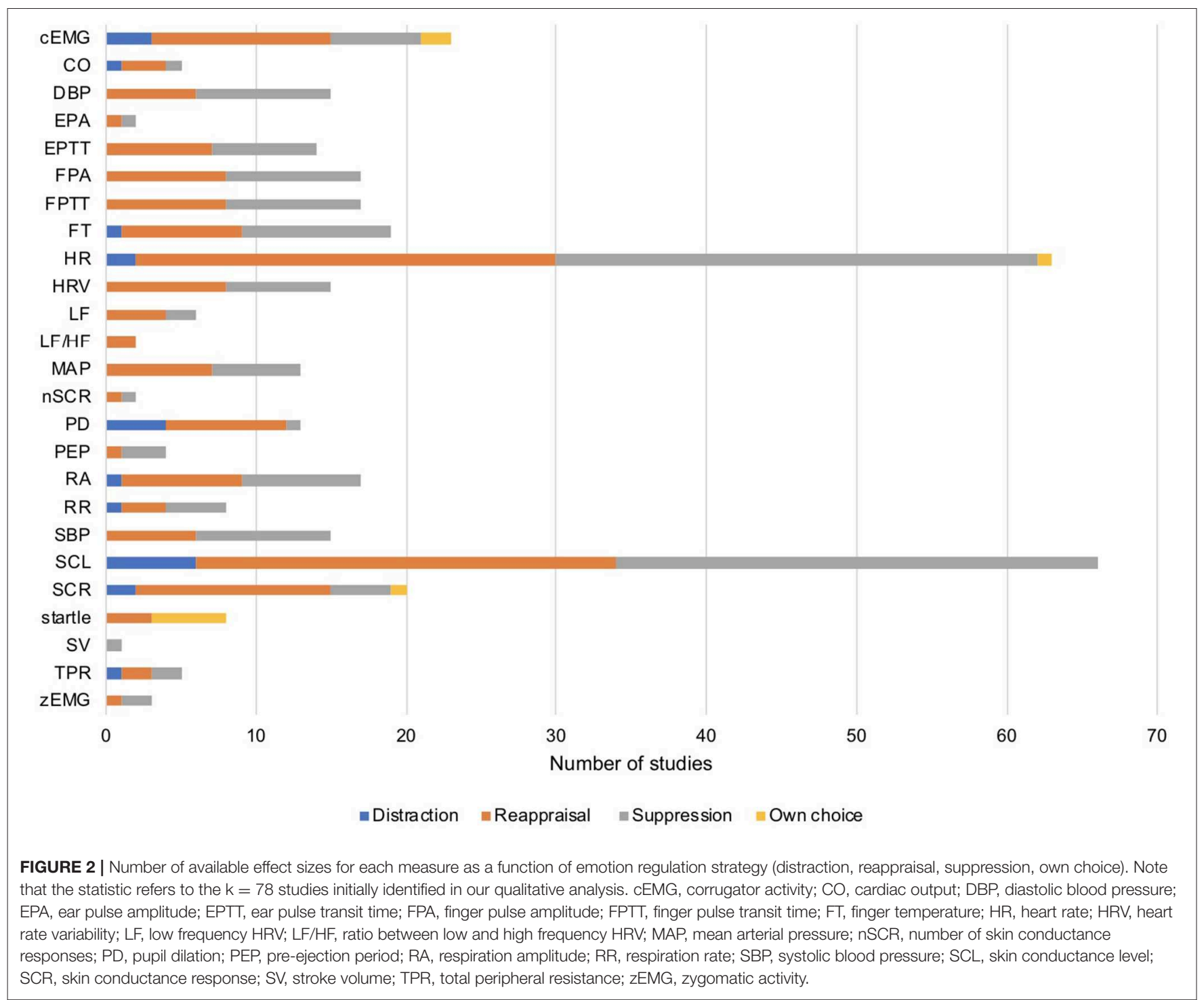

computed mean effect sizes confidence intervals around the mean effect were large (see Figure 3), indicating that the accuracy of our analysis to predict the true effect was rather low. Moreover, heterogeneity differed largely across meta-analyses (see Table 4). For individual forest plots of each meta-analysis see Figures S1-S23.

\section{Cardiovascular Responses}

Reappraisal significantly decreased heart rate $(\mathrm{d}=-0.09, \mathrm{CI}=$ $\left.[-0.17,-0.01], p=0.03, \mathrm{k}=28, I^{2}=21.90\right)$, yet the effect size was very small and direction of effects across individual studies were inconsistent (see Figure S6). Reappraisal had no significant effect on all other tested cardiovascular measures (i.e., finger pulse amplitude, finger pulse transit time, finger temperature, and heart rate variability) with mean effect sizes ranging between -0.02 and 0.16 (see Table 4).

Suppression significantly decreased finger temperature $(\mathrm{d}=$ $-0.33, \mathrm{CI}=[-0.59,-0.07], p=0.02, \mathrm{k}=10, I^{2}=70.03$; see Figure S16), and mean arterial pressure $(\mathrm{d}=-0.34, \mathrm{CI}=$ $[-0.55,-0.12], p=0.01, \mathrm{k}=6, I^{2}=16.45$; see Figure S19), with small to medium effect sizes and mild to notable heterogeneity. Suppression did not significantly change diastolic blood pressure, ear pulse transit time, heart rate, heart rate variability, systolic blood pressure, and skin conductance response (see Table 4 for details and statistics).

\section{Electromyographic Responses}

When considering studies that instructed participants to choose a strategy that worked best for them only, downregulation of negative emotions had a significant negative effect on the emotion-modulated startle $(\mathrm{d}=-0.62, \mathrm{CI}=[-1.02,-0.22]$, $\left.p=0.01, \mathrm{k}=5, I^{2}=47.35\right)^{7}$ with a large effect size and

\footnotetext{
${ }^{7}$ Instructions to downregulate negative emotions (own choice and reappraisal instructions combined) had a significant negative effect on the emotion-modulated startle too $\left(\mathrm{d}=-0.44, \mathrm{CI}=[-0.75,-0.14], p=0.01, \mathrm{k}=8, I^{2}=74.76\right)$.
} 
TABLE 4 | Mean computed effect sizes for each emotion regulation strategy and psychophysiological measure.

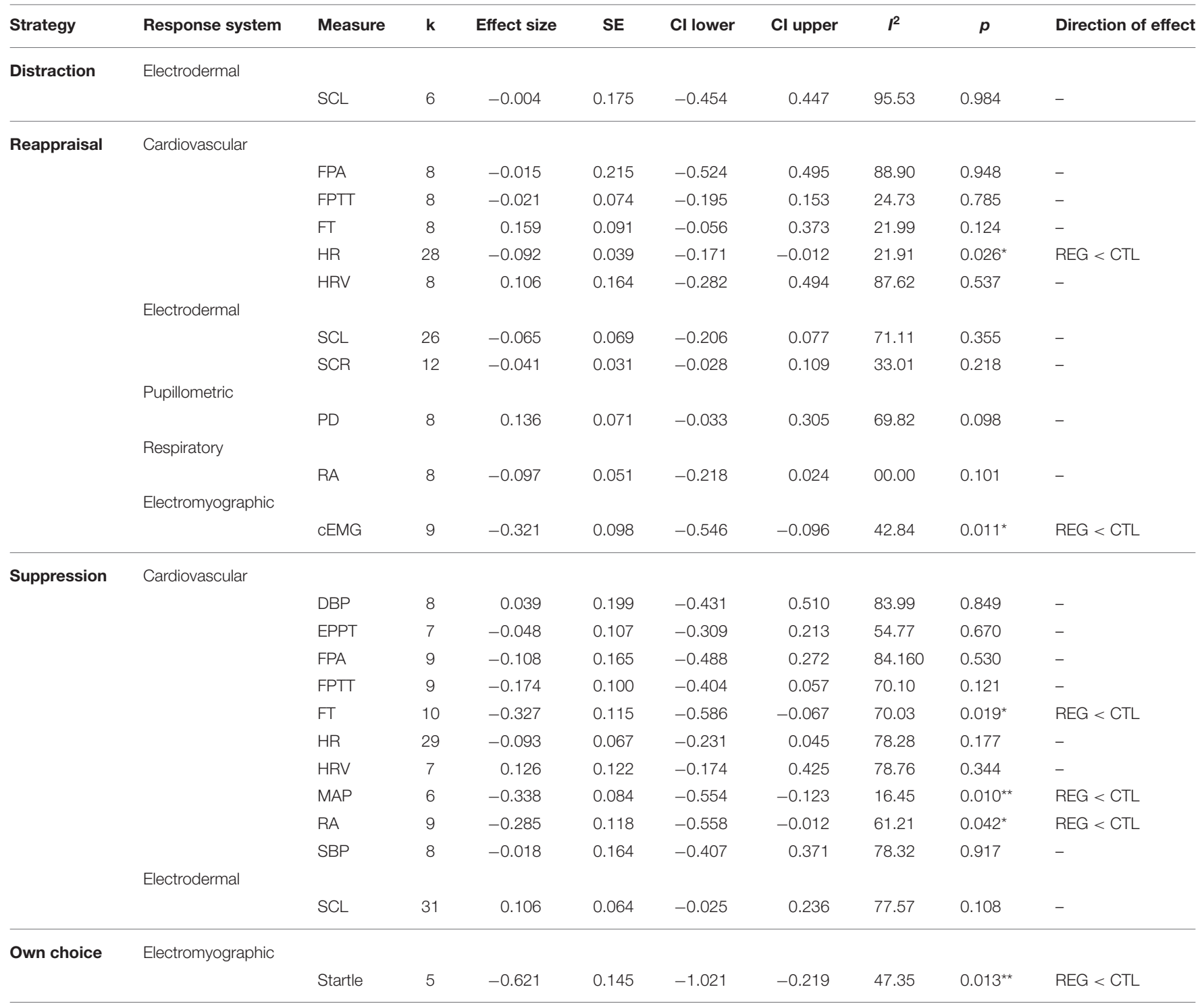

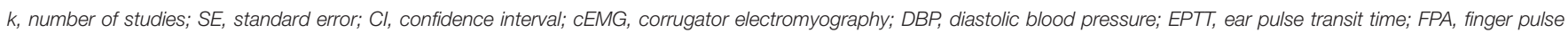

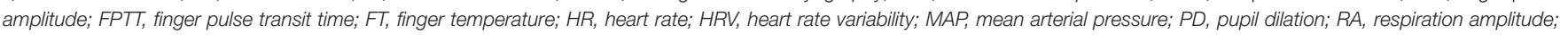

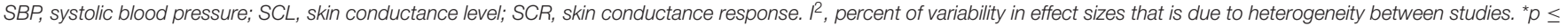
$0.05,{ }^{* \star} p \leq 0.01$.

moderate heterogeneity (see Table 4 and Figure $\mathbf{2 3}$ for details). This means that the instruction to decrease negative emotions reduced, on average, the startle response compared to the control instruction. Moreover, reappraisal significantly decreased corrugator activity $(\mathrm{d}=-0.32, \mathrm{CI}=[-0.55,-0.10], p=0.01$, $\mathrm{k}=9, I^{2}=42.84$ ) with medium effect size and moderate heterogeneity (see Table $\mathbf{4}$ and Figure S2 for details). However, number of studies on the startle $(\mathrm{k}=5)$ and corrugator activity $(\mathrm{k}=9)$ was small and thus should be interpreted with caution.

\section{Electrodermal Responses}

No significant effect was obtained for distraction on skin conductance level compared to the control condition $(\mathrm{d}=$
$-0.004, \mathrm{CI}=[0.98,0.45], p=0.45, \mathrm{k}=6, I^{2}=95.35$; see Figure S1). Similarly, reappraisal had no significant effect on skin conductance level $(\mathrm{d}=-0.07, \mathrm{CI}=[-0.21,0.08], p=0.35, \mathrm{k}=$ 26, $I^{2}=71.11$; see Figure S10) and skin conductance response $\left(\mathrm{d}=0.04, \mathrm{CI}=[-0.03,0.11], p=0.11, \mathrm{k}=12, I^{2}=33.01\right.$; see Figure S11), compared to the control condition.

In addition, suppression did not significantly change the skin conductance level $(\mathrm{d}=0.11, \mathrm{CI}=[-0.03,0.24], p=0.11, \mathrm{k}=31$, $I^{2}=77.57$; see Table 4 and Figure S22).

\section{Respiratory Responses}

Suppression significantly decreased respiration amplitude $(\mathrm{d}=$ -0.29 , CI $[-0.56,-0.01], p=0.04, \mathrm{k}=9, I^{2}=61.21$; see 


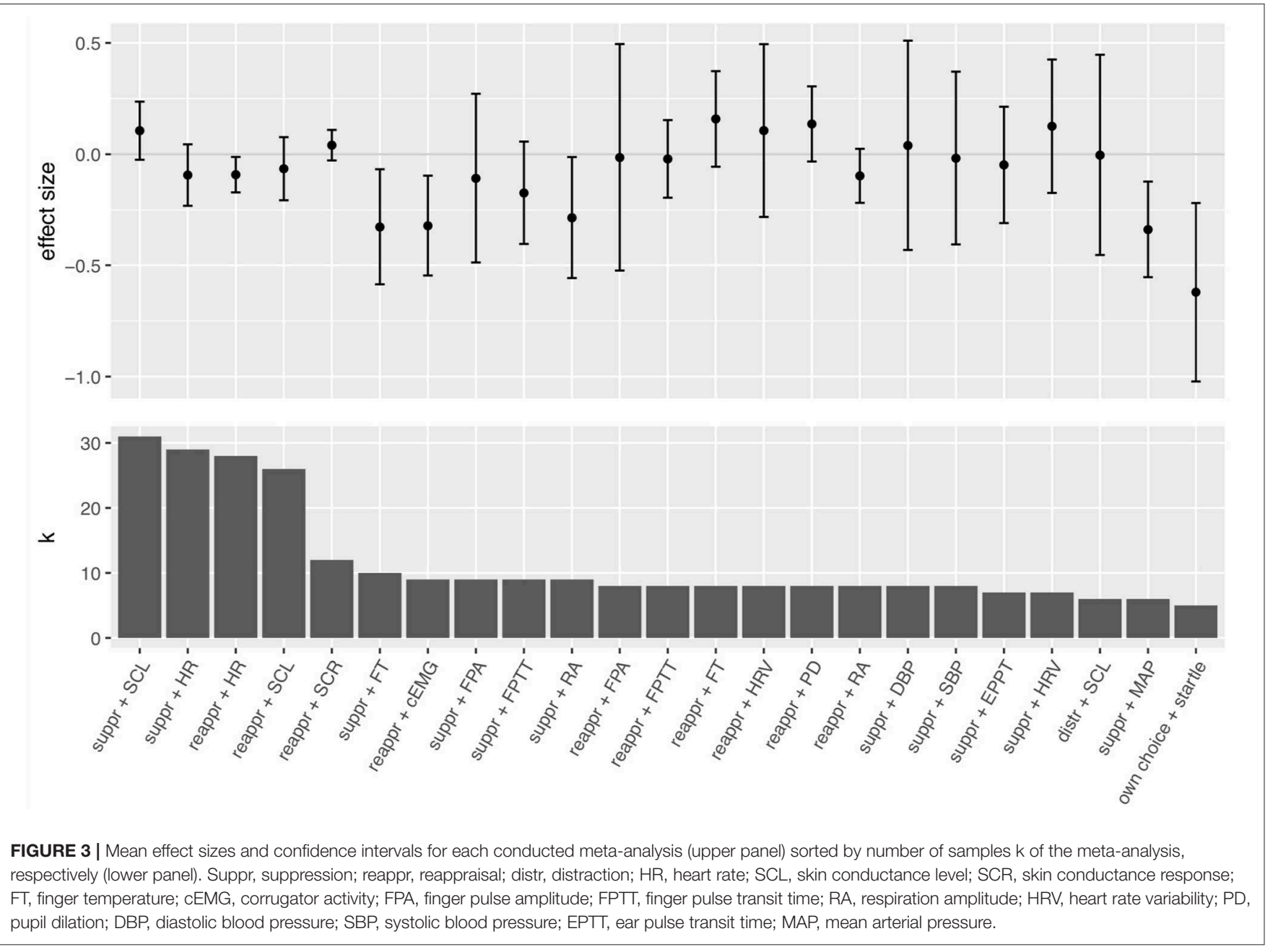

Figure S20). Sample size was small $(\mathrm{k}=9)$ and thus should be interpreted with caution.

\section{Pupillometric Responses}

On average, reappraisal did not significantly change pupil dilation in response to negative stimuli compared to a control condition (see Table 4 and Figure S8 for details). Descriptively, this result might have been driven by one study (Bebko et al., 2011) which found a decrease in pupil size during reappraisal, whereas other studies (van Reekum et al., 2007; Urry et al., 2009; Strauss et al., 2016) found an increase in pupil size during reappraisal. Overall sample size $(\mathrm{k}=8)$ was small and thus should be interpreted with caution.

\section{Evaluation of Publication Bias}

For each significant meta-analysis we constructed a funnel plot with the effect sizes on the horizontal axis and their standard errors on the vertical axis. Egger's tests (Egger et al., 1997) were applied to evaluate asymmetry in funnel plots which may be caused by publication bias. Egger's test revealed that there was significant asymmetry only for the effect of reappraisal on heart rate $(p=0.008)$. Individual funnel plots are presented in the supplement (Figure S24).

\section{Moderator Analyses}

We report moderator analyses only for reappraisal and suppression. For distraction and own choice the number of studies was too small or the distributions of the moderators were inadequate.

\section{Study Design}

Study design (within-subject vs. between-subject) significantly moderated effect sizes of suppression on finger temperature $(\beta$ $=0.54, p \leq 0.01)$, finger pulse amplitude $(\beta=0.78, p \leq 0.001)$, and heart rate $(\beta=-0.38, p \leq 0.01)$. See Table 5 for details. The effect of suppression on finger temperature were significant for between-subject design studies $(\mathrm{d}=-0.62, p \leq 0.001, \mathrm{k}$ $=5$ ), whereas the effect on heart rate became significant for within-subject designs $(\mathrm{d}=-0.29, p \leq 0.001, \mathrm{k}=10)$.

\section{Nature of Control Instruction}

Effect sizes of suppression on finger temperature $(\beta=0.54$, $p \leq 0.01)$, finger pulse transit time $(\beta=0.42, p<0.05)$, 
and finger pulse amplitude $(\beta=0.78, p<0.001)$ were significantly moderated by the control instruction (instruction to respond naturally vs. no instruction) (see Table 6). The effect of suppression on heart rate $(\beta=-0.29, p<0.05)$ and skin conductance level $(\beta=-0.35, p \leq 0.01)$ was also moderated by the control instruction (instruction to respond naturally vs. no instruction). When studies with no instruction were considered only, suppression significantly increased skin conductance level $(\mathrm{d}=0.19, p \leq 0.01, \mathrm{k}=21)$, decreased finger temperature $(\mathrm{d}=-0.62, p \leq 0.001, \mathrm{k}=5)$, and finger pulse transit time $(\mathrm{d}=-0.40, p \leq 0.01, \mathrm{k}=5)$. Conversely, when studies with instruction to respond naturally were considered only, suppression significantly decreased heart rate $(\mathrm{d}=-0.32$, $p \leq 0.01, \mathrm{k}=8)$.

\section{Emotion Induction}

Moderator analyses of effect sizes were conducted for film vs. picture only, as too few studies employing other emotion induction methods for each strategy and psychophysiological

TABLE 5 | Moderator analyses on study design (within-subject design vs. between-subject design).

\begin{tabular}{llccccccc}
\hline Strategy & Measure & $\mathbf{k}$ & $\begin{array}{c}\mathbf{k} \\
\text { (within) }\end{array}$ & $\begin{array}{c}\mathbf{k} \\
\text { (between) }\end{array}$ & $\begin{array}{c}\text { N } \\
\text { total }\end{array}$ & $\boldsymbol{\beta}$ & SE & $\boldsymbol{p}$ \\
\hline Reappraisal & SCL & 26 & 17 & 9 & 1,082 & -0.001 & 0.161 & 0.997 \\
Reappraisal & HR & 28 & 16 & 12 & 1,176 & -0.131 & 0.085 & 0.134 \\
Suppression & SCL & 31 & 11 & 20 & 1,805 & -0.176 & 0.126 & 0.174 \\
Suppression & FT & 10 & 5 & 5 & 701 & 0.543 & 0.138 & $0.004^{* *}$ \\
Suppression & FPTT & 9 & 5 & 4 & 608 & 0.080 & 0.219 & 0.725 \\
Suppression & FPA & 9 & 4 & 5 & 666 & 0.775 & 0.115 & $0.000^{* *}$ \\
Suppression & RA & 9 & 5 & 4 & 467 & -0.188 & 0.263 & 0.497 \\
Suppression & HR & 29 & 10 & 19 & 1,640 & -0.379 & 0.113 & $0.002^{* *}$
\end{tabular}

k, number of studies; SE, standard error; FPA, finger pulse amplitude; FPTT, finger pulse transit time; $F T$, finger temperature; HR, heart rate; $R A$, respiration amplitude; SCL, skin conductance level; $\beta$ regression coefficient (within vs. between). ${ }^{\star \star} p \leq 0.01$.

TABLE 6 | Moderator analyses on nature of control instruction (instruction to respond naturally vs. no instruction).

\begin{tabular}{llccccccc}
\hline Strategy & Measure & $\mathbf{k}$ & $\begin{array}{c}\mathbf{k} \\
\mathbf{C} 4\end{array}$ & $\begin{array}{c}\mathbf{k} \\
\mathbf{C} 1\end{array}$ & $\mathbf{N}$ total & $\boldsymbol{\beta}$ & SE & $\boldsymbol{P}$ \\
\hline Reappraisal & SCL & 23 & 12 & 11 & 986 & -0.063 & 0.127 & 0.625 \\
& SCR & 11 & 7 & 4 & 491 & 0.087 & 0.113 & 0.460 \\
& HR & 25 & 13 & 12 & 1,039 & -0.033 & 0.083 & 0.696 \\
Suppression & SCL & 28 & 7 & 21 & 1,665 & -0.347 & 0.130 & $0.012^{*}$ \\
& FT & 10 & 5 & 5 & 701 & 0.543 & 0.138 & $0.004^{*}$ \\
& FPTT & 9 & 4 & 5 & 608 & 0.422 & 0.156 & $0.030^{*}$ \\
& FPA & 9 & 4 & 5 & 666 & 0.775 & 0.115 & $0.000^{* *}$ \\
& HR & 26 & 8 & 18 & 1,500 & -0.293 & 0.130 & $0.034^{*}$
\end{tabular}

k, number of studies; SE, standard error; FPA, finger pulse amplitude; FPTT, finger pulse transit time; FT, finger temperature; HR, heart rate; SCL, skin conductance level; SCR, skin conductance response; C4, instruction to respond naturally; $C 1$, no instruction; $\beta$ regression coefficient (respond naturally vs. no instruction). ${ }^{*} p \leq 0.05,{ }^{* *} p \leq 0.01$. measure combination were available to interpret moderator analyses in a meaningful way. Emotion induction (films vs. pictures) did not significantly moderate the effect sizes of reappraisal and suppression on skin conductance level and heart rate (see Table 7).

\section{Trial Duration}

Trial duration significantly moderated the effect of reappraisal on skin conductance response $(\beta=-0.03, p=0.05, \mathrm{k}=12)$ and the effect of suppression on skin conductance level $(\beta=-0.03, p<$ $0.05, \mathrm{k}=31)$, diastolic $(\beta=-0.41, p<0.05, \mathrm{k}=8)$ and systolic blood pressure ( $\beta=-0.39, p<0.01, \mathrm{k}=8)$ in that the effect became more negative with longer trial durations (see Table 8). The moderating effect of trial duration on suppression and skin conductance level was mainly driven by one study (Yuan et al., 2014).

TABLE 7 | Moderator analyses on emotion induction (films vs. pictures).

\begin{tabular}{lllllllll}
\hline Strategy & Measure & $\mathbf{k}$ & $\begin{array}{c}\mathbf{k} \\
\text { films }\end{array}$ & $\begin{array}{c}\mathbf{k} \\
\text { pictures }\end{array}$ & $\begin{array}{c}\text { N } \\
\text { total }\end{array}$ & $\boldsymbol{\beta}$ & SE & $\boldsymbol{p}$ \\
\hline Reappraisal & SCL & 23 & 16 & 7 & 900 & 0.126 & 0.167 & 0.458 \\
Reappraisal & HR & 20 & 12 & 8 & 723 & -0.150 & 0.086 & 0.101 \\
Suppression & SCL & 26 & 22 & 4 & 1,431 & 0.049 & 0.187 & 0.795 \\
Suppression & HR & 25 & 19 & 6 & 1,256 & 0.145 & 0.144 & 0.324 \\
\hline
\end{tabular}

k, number of studies; SE, standard error; HR, heart rate; SCL, skin conductance level; $\beta$, regression coefficient (films vs. pictures).

TABLE 8 | Moderator analyses on trial duration.

\begin{tabular}{llccccc}
\hline Strategy & Measure & $\mathbf{k}$ & $\mathbf{N}$ total & $\boldsymbol{\beta}$ & SE & $\boldsymbol{p}$ \\
\hline Distraction & SCL & 6 & 287 & 0.084 & 0.081 & 0.354 \\
Reappraisal & HR & 28 & 1176 & 0.015 & 0.012 & 0.209 \\
& HRV & 8 & 305 & 0.071 & 0.053 & 0.232 \\
& PD & 8 & 250 & -2.492 & 1.996 & 0.258 \\
& SCL & 26 & 1,082 & 0.021 & 0.021 & 0.324 \\
& SCR & 12 & 530 & -0.028 & 0.013 & $0.053^{*}$ \\
Suppression & CEMG & 9 & 354 & 0.000 & 0.051 & 0.997 \\
& DBP & 8 & 440 & -0.408 & 0.141 & $0.028^{*}$ \\
& EPPT & 7 & 551 & 0.022 & 0.024 & 0.403 \\
& FPA & 9 & 666 & -0.030 & 0.039 & 0.464 \\
& FPTT & 9 & 608 & -0.011 & 0.026 & 0.677 \\
& FT & 10 & 701 & 0.130 & 0.086 & 0.172 \\
& HR & 29 & 1640 & 0.028 & 0.022 & 0.214 \\
& HRV & 7 & 491 & 0.044 & 0.047 & 0.392 \\
& RA & 9 & 467 & -0.032 & 0.048 & 0.526 \\
& SBP & 8 & 440 & -0.387 & 0.094 & $0.006^{* *}$ \\
& SCL & 31 & 1,805 & -0.026 & 0.012 & $0.039^{*}$
\end{tabular}

k, number of studies; SE, standard error; CEMG, corrugator electromyography; DBP, diastolic blood pressure; EPTT, ear pulse transit time; FPA, finger pulse amplitude; FPTT, finger pulse transit time; FT, finger temperature; HR, heart rate; HRV, heart rate variability; MAP, mean arterial pressure; $P D$, pupil dilation; $R A$, respiration amplitude; $S B P$, systolic blood pressure; SCL, skin conductance level; SCR, skin conductance response; $\beta$ regression coefficient (refers to 1 min change in trial duration). ${ }^{*} p \leq 0.05,{ }^{* *} p \leq 0.01$. 


\section{DISCUSSION}

Over the past two decades, emotion regulation has become a vibrant research field. Our literature search corroborates this trend. It revealed an increase of almost $60 \%$ of potentially relevant publications for our meta-analysis within the recent 3 years. The vast growth of literature illustrates a vigorous interest in understanding the psychophysiological mechanisms of emotion regulation.

Previous studies on the psychophysiological responses to emotion regulation revealed inconsistent results. Moreover, distraction and reappraisal strategies appeared to have no or little effect on psychophysiology (Webb et al., 2012), and suppression significantly increased sympathetic arousal (Gross and Levenson, 1993; Gross, 1998a). This meta-analysis provides the first attempt to elucidate common trends with means of a quantitative summary of the effects of common emotion regulation strategies on different cardiovascular, electrodermal, respiratory, pupillometric, and electromyographic measures. We performed a structured literature review and conducted a metaanalysis for each combination of psychophysiological measure and emotion regulation strategy whenever there were enough studies available. In brief, we found that suppression significantly decreased mean arterial pressure, finger temperature, and respiration amplitude, whereas reappraisal led to decreased heart rate and decreased corrugator activity (see Table 4 and Figure 3 for an overview of effects). When participants were free to choose between emotion regulation strategies, a significant inhibition of the emotion-modulated startle (sometimes referred to as fearpotentiated startle) response could be observed. Due to the limited number of studies on distraction, we were not able to conduct meta-analyses on psychophysiological responses except for skin conductance level, and this meta-analysis revealed no significant effect. Publication bias appeared to have an overall minor effect.

As Figure 3 illustrates, aggregated effect sizes from the tested autonomic responses were small in general. We did not compute an overall effect size across all psychophysiological measures. Yet aggregated effect sizes for each psychophysiological measure correspond with the results reported by Webb et al.'s metaanalysis (Webb et al., 2012). They had reported an overall small negative effect of response modulation (e.g., suppression strategies) on psychophysiology $(d=0.19,[\mathrm{CI}=0.14,0.01])$. Attentional deployment (e.g., distraction strategies) had no significant effect on physiological measures $(d=0.00, \mathrm{CI}=$ $[0.14,0.15])$, and so did cognitive change (e.g., reappraisal) $(d$ $=0.05$, $[\mathrm{CI}=0.07$ to 0.16$]$ ) (Webb et al., 2012). We conclude that effects of emotion regulation on autonomic measures-if at all present-seem to be rather small and raise the question whether emotion regulation success can be reliably quantified with autonomic measures. It should however be noted that the psychophysiological measures entering our analysis were limited. Figure 2 illustrates that there were a number of measures not included as too few studies were available. For example, measures of cardiac function that can be derived via impedance cardiography have received scant attention in the previous literature but provide promising results: Studies have shown that emotion regulation changed total peripheral resistance with medium to large effect sizes (Jamieson et al., 2012, 2013; Peters et al., 2014; Peters and Jamieson, 2016).

Activation of the sympathetic nervous system causes an increase in skin conductivity, pupil dilation, heart rate, preejection period, blood pressure, peripheral vasoconstriction, and increased respiration amplitude and respiration rate. Successful emotion regulation should be accompanied by a reduction of sympathetic activity (McRae and Shiota, 2017). Our study reveals that the effects are not quite that straightforward. Suppression lowered finger temperature (indicative of increased sympathetic activity), yet also decreased mean arterial pressure and respiration amplitude (indicative of lower sympathetic activity). Similarly, reappraisal decreased heart rate (indicative of lower sympathetic activity) but did not change any of the other tested autonomic measures. McRae and Shiota (2017) point out that psychophysiological effects often diverge in patterns that correspond to different psychological states (Kreibig, 2010; Shiota et al., 2011), which can result in misinterpretations about the association between psychophysiological responses and the underlying psychological processes (Cacioppo and Tassinary, 1990; Cacioppo et al., 2007). Psychophysiological responses are usually influenced by various factors, such as stress, workload, or tiredness, and thus may distort the effects of emotion regulation. Decreased pupil size during reappraisal was observed in one study and has been interpreted to be the result of decreased emotional arousal (Bebko et al., 2011). Alternatively, studies have interpreted larger pupil size during reappraisal as an indicator of higher cognitive effort (Urry et al., 2006; van Reekum et al., 2007). They infer that pupil size may increase during successful emotion regulation as an indicator of increased cognitive processing. The ambiguity of such effects implies that we need a better understanding of cognitive and emotional processes causing autonomic change, and how these changes relate to emotion regulation success.

Another problem is the inconsistency of direction of effect sizes. Different directions of effect sizes rendered the metaanalyses insignificant and infer that there are important factors not yet understood. For example, the meta-analysis of pupil dilation during reappraisal (see Figure S8) revealed that one study (Bebko et al., 2011), which received a strong weight in the analysis, found a significant decrease in pupil diameter during reappraisal, while other studies found an increase in pupil diameter (e.g., van Reekum et al., 2007; Urry et al., 2009; Strauss et al., 2016). Similarly, our meta-analysis on heart rate during suppression (see Figure S17) revealed that studies found mean heart rate acceleration in response to suppression (e.g., Hagemann et al., 2006; Stiller et al., 2019), whereas other studies found a heart rate deceleration (Kunzmann et al., 2005; DanGlauser and Gross, 2011, 2015). Therefore, the second aim of the present work was to explore the impact of methodological differences using several moderators (trial duration, nature of emotion induction, nature of control instruction, study design).

Effects of suppression on heart rate, finger temperature and finger pulse amplitude were significantly moderated by study design (within vs. between-subject). Between-subject design studies showed a significant decrease in finger temperature and 
finger pulse amplitude during suppression whereas studies with a within-subject design revealed no significant effect. Conversely, within-subject design studies showed a significant decrease in heart rate whereas studies with a between-subject design revealed no significant effect. The moderating effect of study design on heart rate might also reflect that between-subject design studies in this particular meta-analysis assessed extremely diverse emotion induction methods. For example, two studies (Butler et al., 2006; Ben-Naim et al., 2013) assessed emotion regulation in dyadic interactions. Hagemann et al. (2006) used startle tones in combination with pictures. Rohrmann et al. (2009), Gross (1998a), Denson et al. (2011) used film stimuli. Within-subject design studies considered in this meta-analysis used films and pictures only. Therefore, the nature of emotion induction may account for some variance in the effect sizes obtained across studies using between-subject designs. When data from more studies will be available in the future, it might be possible to confirm this assumption.

Effects of reappraisal and suppression on several electrodermal and cardiovascular measures (i.e., skin conductance level, finger temperature, finger pulse transit time, finger pulse amplitude, and heart rate) were significantly moderated by the nature of control instructions. Except for finger pulse amplitude, the effects became significant when no instruction (i.e., "view" instruction) was given but did not become significant when the instruction to respond naturally was given. This does not correspond with findings by Webb et al. (2012) who found that emotion regulation strategies in general had smaller effects on experiential, behavioral and physiological measures combined when the control condition required participants to "view" or "not to regulate" and larger effects when the control condition required participants to respond naturally. In contrast to our study, they did not determine the moderating effect of control instruction on physiological effects of emotion regulation but considered the overall effect of psychophysiological, behavioral and experiential measures. Control conditions requiring participants to simply view a negative stimulus might correspond to a physiological baseline condition. However, when receiving the instruction to respond naturally, participants might unconsciously pay more attention to their emotional response, which may be particularly sensitive to psychophysiological responses.

Trial duration significantly moderated effect sizes of suppression on skin conductance level, diastolic and systolic blood pressure, and of reappraisal on skin conductance response in that the effects became more negative with increasing trial length. Studies on electrodermal responses may be difficult to compare within the conducted meta-analyses because trial durations varies largely across studies. This might be especially problematic for skin conductance level, as longer time windows carry the risk that non-specific skin conductance responses occur. If these phasic responses are not separated from the tonic parts, they might influence the absolute skin conductance level (Boucsein et al., 2012). Hence, skin conductance level assessed over several seconds in an event-related design might be different than skin conductance level assessed over several minutes in a block-design. We accounted for this variability in parts by conducting a moderator analysis with trial duration as the moderator. We observed effects in both positive and negative direction. Studies with very short trial duration tend to report an increase in skin conductance, whereas studies with longer or extremely long trial durations tend to report a decrease in skin conductance. However, we acknowledge that our analysis did not allow to differentiate for example between studies that assessed skin conductance averages but eliminated the tonic parts (Hallam et al., 2015; Plieger et al., 2017) and studies that assessed skin conductance level without separating the phasic from the tonic responses. We encourage future researcher to use similar research methodology and terminology as suggested by the committee report on publication recommendations (Boucsein et al., 2012) to make studies more comparable in the future. In total, the varying effects of skin conductance across studies may be in part due to the high variability in assessment and quantification.

Compared to the tested autonomic responses (i.e., cardiovascular, electrodermal, pupillometric and respiratory responses), our present analysis revealed that effects of measures assessed with electromyography were medium and consistent across individual studies (see Figures S2, S23). Regarding the emotion-modulated startle, we found a significant decrease through emotion downregulation with a mean effect size of $\mathrm{d}=-0.62$. Corrugator activity significantly decreased with reappraisal of negative emotions with a medium effect size of $d$ $=-0.32$. As both analyses included a rather small number of studies resulting in large confidence intervals, they should be treated with caution (see Figure 3). Nevertheless, the results on electromyography showed more consistent results compared to the autonomic measures assessed in the present review and this encourages possible reasons that might have accounted for this consistency.

Studies have shown that both the emotion-modulated startle and corrugator activity are specific to valence: The startle is inhibited in response to pleasant but potentiated in response to unpleasant stimuli with stronger responses for high- than for low-arousing stimuli (Vrana et al., 1988; Bradley et al., 1993a; Hamm et al., 1997; Schupp et al., 1997; Hawk and Cook, 2000). Corrugator supercilii is generally considered to correspond to changes in valence, too (Tassinary et al., 2007). The valencespecificity might facilitate to measure the correspondence to changes in valence and hence allows to track the regulation effect more closely, compared with autonomic measures that rather reflect changes in arousal. However, there are also studies showing that in the context of emotion regulation, the startle response is more sensitive to changes in arousal (Dillon and LaBar, 2005; Zaehringer et al., 2018).

Animal studies have shown that the amygdala, a key structure in emotion processing, directly modulates the auditory startle reflex via modulation of midbrain neurons (Rosen and Davis, 1988; Davis, 1992), which has been recently complemented by fMRI work in human subjects (Kuhn et al., 2019). Researcher have argued that the emotional modulation as indexed by the startle reflex may serve as a direct indicator of amygdala activation independent of task demands (Grillon and Baas, 2003). Similarly, the amygdala projects to the facial motor 
nucleus thereby coupling emotional facial expressions to the motive circuit (Davis, 2000). The amygdala is a robust neural target of emotion regulation (Buhle et al., 2014) and altered amygdala activation with emotion regulation thus likely mediates the modulatory effect on the startle response and corrugator activity. Taken together, the specificity to the valence dimension and the direct modulation via the brain's motivational system may contribute to the findings of emotion regulation effects on emotion modulated startle and corrugator activity.

With regard to the emotion-modulated startle, it is also possible that the emotion regulation instruction might have influenced the obtained effect sizes. Participants in these studies were free to choose an emotion regulation strategy that worked best for them. By allowing participants to choose from different strategies, they might be more successful in regulating their emotions, which could result in larger effects. Moreover, the startle response unfolds within milliseconds, whereas autonomic responses such as pupil dilation, electrodermal responses, and heart rate variability rather unfold over several seconds, or even minutes. Therefore, the startle response may be easier to measure because it is clearly time-locked to the startle probe and all changes can be measured in studies with shorter observation times during the trials, whereas a skin conductance response with a slower response latency to peak may carry over effects to the next trial. In addition, emotion-modulated startle studies largely converge on the measurement and quantification of the startle response, whose setup is known to be relatively simple. In our meta-analysis on the emotion-modulated startle, all studies rectified and integrated the raw EMG signal with a time constant of $20 \mathrm{~ms}$, calculated the startle amplitude by subtracting a 20 or $50 \mathrm{~ms}$ pre-startle baseline from the peak $20-120$ or $20-150 \mathrm{~ms}$ after startle probe onset and finally t- or z-transformed the mean amplitudes (Jackson et al., 2000; Dillon and LaBar, 2005; Golkar et al., 2014; Conzelmann et al., 2015).

In contrast, we observed tremendous variation in the quantification of the autonomic indices. For example, studies on skin conductance level during reappraisal assessed baseline activity during a neutral condition that included the presentation of neutral stimuli (Wolgast et al., 2011; Lohani and Isaacowitz, 2014), right before stimulus onset (e.g., Shiota and Levenson, 2009), right before instruction (Opitz et al., 2014), after instruction (Urry et al., 2009), or reported no baseline assessment (Goldin et al., 2019). These studies then either subtracted mean activity of the respective baseline from mean activity during the regulation period (e.g., Shiota and Levenson, 2009; Opitz et al., 2014), calculated raw means (Goldin et al., 2019), or area under the curve (Urry et al., 2009). It should be noted that these observations remain solely on a descriptive level. We did not conduct a moderator analysis to account for this variation since too few studies were available. Future studies would be helpful to corroborate our considerations.

The meta-analyses we presented in this article suggest that electromyographic measures such as the emotion-modulated startle might be robust options to assess emotion regulation effects, whereas autonomic measures might be context dependent and thus should be selected carefully. Autonomic measures are still important and interesting for emotion regulation research as they allow to track the extended reaction of the body to an emotional event or a series of events, whereas the emotion-modulated startle is being assessed at one given time and thus does not allow to track the time-course of the regulation period.

\section{Limitations and Future Research}

While the present study represents the first meta-analysis of specific psychophysiological effects during distraction, reappraisal, suppression, and instructions to choose a downregulation strategy, it is not without limitations. First of all, we emphasize that the number of available studies was small with the exception of heart rate and skin conductance level. In particular, most of the significant meta-analyses in the present study included few studies and these studies often stemmed from an even smaller number of labs (e.g., mean arterial pressure, finger temperature; see Figure 3). Thus, we need more research to test whether the effects would become insignificant with increasing number of independent studies. Similarly, absence of significance in meta-analyses with small number of samples should not be taken as evidence that there is no effect at all. Thus, studies that assess less common psychophysiological measures and emotion regulation instructions are urgently needed to increase knowledge about psychophysiological responses during emotion regulation.

Furthermore, no meta-analysis is free of a potential publication bias. The bias refers to the phenomenon that significant findings get published earlier and are more likely than non-significant findings. Statistical analyses indicated that there might be some publication bias, but this seemed not to appreciably impact the results. In addition, psychophysiological measures are usually not the primary outcome of emotion regulation studies, and many published studies have reported negative findings. Thus, we consider the publication bias to be relatively small in this review.

We also highlight the substantial variability in the research methodology used across the emotion regulation studies included in our meta-analysis. We explored the impact of methodological differences using several moderators (trial duration, nature of emotion induction, nature of control instruction, study design) and showed that central design aspects are explaining some differences in the overserved autonomic effect sizes. This raises the question to which degree the studies included in the present review are actually comparable.

Sample size was very small and conducting the meta-analyses and moderator analyses required a large number of separate analyses. In light of this, significant results presented here should be treated with caution as multiple comparisons might have increased the chances of false discovery. More research is needed to confirm our results. We also acknowledge that we assessed a limited sample of potential moderators. As mentioned above, there was tremendous variation in the quantification of the autonomic indices, which we were not able to account for as there were too few studies available to conduct meaningful moderator analyses. Finally, we highlight that our meta-analysis was limited to the regulation of negative emotions only, mainly focusing on reappraisal and suppression. 
In light of these limitations, we need particularly larger and more comparable studies with identical setup to control the moderator variables identified in this meta-analysis (in particular trial duration, comparable control conditions and the same study design). One important future direction for researchers in the area of psychophysiological response patterns to emotion regulation is to design large-scale, comprehensive studies that directly compare psychophysiological measures and emotion regulation strategies ideally using the same assessment and quantification of psychophysiological responses.

With psychophysiological recordings we cannot control which regulation strategies are really being applied by participants. The variability of autonomic responding across different emotion regulation contexts further complicates an accurate interpretation of effects and may be particularly problematic in studies focusing on just one psychophysiological outcome measure. Experiments using simultaneous recordings from multiple psychophysiological channels would be helpful to e.g., identify potential response patterns uniquely characterizing different emotion regulation strategies (e.g., pupil, heart rate, skin conductance, etc.). However, major progress is unlikely without coordinated effort across labs to systematically address these questions.

There is also a need for studies that carefully tease apart attention, arousal and other cognitive processes that may influence autonomic responses in order to gain a better understanding of the interpretation of autonomic responses during emotion regulation. Systematic variations in different experimental setups may help to dissociate the underlying cognitive and emotional processes that cause autonomic activity in order to draw clear inferences.

\section{CONCLUSION}

This meta-analysis represents the first attempt to determine the mean effects of different emotion regulation strategies on individual psychophysiological measures. Our results indicate that (a) effects of reappraisal decreased heart rate and corrugator activity, whereas suppression increased sympathetic arousal but decreased respiration amplitude and mean arterial pressure, (b) effects of autonomic measures, even if significant, were small and heterogeneous across studies, while electromyographic measures showed medium effect sizes and (c) the study design, control instruction and trial duration moderated some but not all effect sizes. As available studies were few, our findings remain preliminary. In order to use meta-analyses to

\section{REFERENCES}

Ajaya, Y., Peckham, A. D., and Johnson, S. L. (2016). Emotion regulation and mania risk: differential responses to implicit and explicit cues to regulate. J. Behav. Ther. Exp. Psychiatry 50, 283-288. doi: 10.1016/j.jbtep.2015. 10.004

Aldao, A., and Mennin, D. S. (2012). Paradoxical cardiovascular effects of implementing adaptive emotion regulation strategies in generalized compare effects of psychophysiological responses in different regulation contexts, more comparable methodological set-ups should be used in the empirical study of emotion regulation. The induction of specific types of emotions and the assessment of less common psychophysiological measures and regulation strategies will allow future meta-analyses to fully discover the potential influences on psychophysiological response during emotion regulation.

\section{DATA AVAILABILITY STATEMENT}

All datasets generated for this study are included in the article/Supplementary Material.

\section{AUTHOR CONTRIBUTIONS}

JZ, CP, CS, and GE were involved in the conception of the work. JZ planned and conducted the literature search, coded the data and together with CJ-S designed and carried out the data analysis. JZ drafted the manuscript. CP, CJ-S, CS, and GE revised it critically for important intellectual content.

\section{FUNDING}

This research was supported by the German Research Foundation (grant no. KFO 256, EN 361/13-2) and by a scholarship from the German Bischöfliche Studienförderung Cusanuswerk awarded to JZ. We also acknowledge financial support by Deutsche Forschungsgemeinschaft within the funding programme Open Access Publishing, by the Baden-Württemberg Ministry of Science, Research and the Arts and by Ruprecht-Karls-Universität Heidelberg.

\section{ACKNOWLEDGMENTS}

We thank all the authors who kindly provided additional data and statistics. Furthermore, we thank Stephanie Mall for help with the screening and coding of studies and Janick Frasch for his help with creating the figures.

\section{SUPPLEMENTARY MATERIAL}

The Supplementary Material for this article can be found online at: https://www.frontiersin.org/articles/10.3389/fpsyg. 2020.00470/full\#supplementary-material strategies across psychopathology: a meta-analytic review. Clin. Psychol. Rev. 30, 217-237. doi: 10.1016/j.cpr.2009.11.004

Alpers, G. W., Adolph, D., and Pauli, P. (2011). Emotional scenes and facial expressions elicit different psychophysiological responses. Int. J. Psychophysiol. 80, 173-181. doi: 10.1016/j.ijpsycho.2011.01.010 
Azbel-Jackson, L., Butler, L. T., Ellis, J. A., and van Reekum, C. M. (2015). Stay calm! Regulating emotional responses by implementation intentions: assessing the impact on physiological and subjective arousal. Cogn. Emot. 30, 1107-1121. doi: 10.1080/02699931.2015.1049515

Baur, R., Conzelmann, A., Wieser, M. J., and Pauli, P. (2015). Spontaneous emotion regulation: differential effects on evoked brain potentials and facial muscle activity. Int. J. Psychophysiol. 96, 38-48. doi: 10.1016/j.ijpsycho.2015.02.022

Bebko, G. M., Franconeri, S. L., Ochsner, K. N., and Chiao, J. Y. (2011). Look before you regulate: differential perceptual strategies underlying expressive suppression and cognitive reappraisal. Emotion 11, 732-742. doi: 10.1037/a0024009

Ben-Naim, S., Hirschberger, G., Ein-Dor, T., and Mikulincer, M. (2013). An experimental study of emotion regulation during relationship conflict interactions: the moderating role of attachment orientations. Emotion 13, 506-519. doi: 10.1037/a0031473

Bernat, E. M., Cadwallader, M., Seo, D., Vizueta, N., and Patrick, C. J. (2011). Effects of instructed emotion regulation on valence, arousal, and attentional measures of affective processing. Dev. Neuropsychol. 36, 493-518. doi: 10.1080/87565641.2010.549881

Berntson, G. G., Quigley, K. S., Norman, G. J., and Lozano, D. L. (2016). "Cardiovascular psychophysiology," in Handbook of Psychophysiology, 4th Edn, eds J. T. Cacioppo, L. G. Tassinary, and G. G. Berntson (Cambridge: Cambridge University Press), 183-216.

Blumenthal, T. D., Cuthbert, B. N., Filion, D. L., Hackley, S., Lipp, O. V., and van Boxtel, A. (2005). Committee report: guidelines for human startle eyeblink electromyographic studies. Psychophysiology 42, 1-15. doi: 10.1111/j.1469-8986.2005.00271.x

Borenstein, M., Hedges, L. V., Higgins, J. P., and Rothstein, H. R. (2010). A basic introduction to fixed-effect and random-effects models for meta-analysis. Res. Synth. Methods 1, 97-111. doi: 10.1002/jrsm.12

Boucsein, W., Fowles, D. C., Grimnes, S., Ben-Shakhar, G., Roth, W. T., Dawson, M. E., et al. (2012). Publication recommendations for electrodermal measurements. Psychophysiology 49, 1017-1034. doi: 10.1111/j.1469-8986.2012.01384.x

Braams, B. R., Blechert, J., Boden, M. T., and Gross, J. J. (2012). The effects of acceptance and suppression on anticipation and receipt of painful stimulation. J. Behav. Ther. Exp. Psychiatry 43, 1014-1018. doi: 10.1016/j.jbtep.2012. 04.001

Bradley, M. M., Codispoti, M., Cuthbert, B. N., and Lang, P. J. (2001). Emotion and motivation I: defensive and appetitive reactions in picture processing. Emotion 1, 276-298. doi: 10.1037/1528-3542.1.3.276

Bradley, M. M., Cuthbert, B. N., and Lang, P. J. (1993a). Pictures as prepulse: attention and emotion in startle modification. Psychophysiology 30, 541-545. doi: 10.1111/j.1469-8986.1993.tb02079.x

Bradley, M. M., and Lang, P. J. (2000). "Measuring emotion: behavior, feeling, and physiology," in Cognitive Neuroscience of Emotion, eds R. D. Lane, L. Nadel, R. D. Lane, and L. Nadel (New York, NY: Oxford University Press), 242-276.

Bradley, M. M., Lang, P. J., and Cuthbert, B. N. (1993b). Emotion, novelty, and the startle reflex: habituation in humans. Behav. Neurosci. 107, 970-980. doi: 10.1037/0735-7044.107.6.970

Buhle, J. T., Silvers, J. A., Wager, T. D., Lopez, R., Onyemekwu, C., Kober, H., et al. (2014). Cognitive reappraisal of emotion: a meta-analysis of human neuroimaging studies. Cereb. Cortex 24, 2981-2990. doi: 10.1093/cercor/bht154

Bulut, N. S., Würz, A., Küpeli, N. Y., Bulut, G. Ç., and Sungur, M. Z. (2018). Heart rate variability response to affective pictures processed in and outside of conscious awareness: three consecutive studies on emotional regulation. Int. J. Psychophysiol. 129, 18-30. doi: 10.1016/j.ijpsycho.2018.05.006

Butler, E. A., Egloff, B., Wlhelm, F. H., Smith, N. C., Erickson, E. A., and Gross, J. J. (2003). The social consequences of expressive suppression. Emotion 3, 48-67. doi: 10.1037/1528-3542.3.1.48

Butler, E. A., Gross, J. J., and Barnard, K. (2014). Testing the effects of suppression and reappraisal on emotional concordance using a multivariate multilevel model. Biol. Psychol. 98, 6-18. doi: 10.1016/j.biopsycho.2013.09.003

Butler, E. A., Wilhelm, F. H., and Gross, J. J. (2006). Respiratory sinus arrhythmia, emotion, and emotion regulation during social interaction. Psychophysiology 43, 612-622. doi: 10.1111/j.1469-8986.2006.00467.x

Cacioppo, J. T., Berntson, G. G., Larsen, J. T., Poehlmann, K. M., and Ito, T. A. (2000). "The psychophysiology of emotion," in Handbook of Emotions,
Vol. 2, eds M. Lewis and J. M. Haviland-Jones (New York, NY: Guilford Press), 173-191.

Cacioppo, J. T., and Tassinary, L. G. (1990). Inferring psychological significance from physiological signals. Am. Psychol. 45:16.

Cacioppo, J. T., Tassinary, L. G., and Berntson, G. G. (2007). Handbook of Psychophysiology, Vol. 2. New York, NY: Cambridge University Press.

Chu, V. C., Lucas, G. M., Lei, S., Mozgai, S., Khooshabeh, P., and Gratch, J. (2019). Emotion regulation in the Prisoner's Dilemma: effects of reappraisal on behavioral measures and cardiovascular measures of challenge and threat. Front. Hum. Neurosci. 13:50. doi: 10.3389/fnhum.2019.00050

Cohen, J. E. (1988). Statistical Power Analysis for the Behavioral Sciences. Hillsdale, NJ: Lawrence Erlbaum Associates, Inc.

Colby, C. Z., Lanzetta, J. T., and Kleck, R. E. (1977). Effects of the expression of pain on autonomic and pain tolerance responses to subject-controlled pain. Psychophysiology 14, 537-540. doi: 10.1111/j.1469-8986.1977.tb01194.x

Conzelmann, A., McGregor, V., and Pauli, P. (2015). Emotion regulation of the affect-modulated startle reflex during different picture categories. Psychophysiology 52, 1257-1262. doi: 10.1111/psyp.12450

Dan-Glauser, E. S., and Gross, J. J. (2011). The temporal dynamics of two response-focused forms of emotion regulation: experiential, expressive, and autonomic consequences. Psychophysiology 48, 1309-1322. doi: 10.1111/j.1469-8986.2011.01191.x

Dan-Glauser, E. S., and Gross, J. J. (2015). The temporal dynamics of emotional acceptance: experience, expression, and physiology. Biol. Psychol. 108, 1-12. doi: 10.1016/j.biopsycho.2015.03.005

Davis, M. (1992). The role of the amygdala in fear-potentiated startle: implications for animal models of anxiety. Trends Pharmacol. Sci. 13, 35-41. doi: 10.1016/0165-6147(92)90014-W

Davis, M. (2000). "The role of the amygdala in conditioned and unconditioned fear and anxiety," in The Amygdala, Vol. 2, ed J. P. Aggleton (Oxford: Oxford University Press), 213-287.

Dawson, M. E., Schell, A. M., and Filion, D. L. (2016). “The electrodermal system," in Handbook of Psychophysiology, 4th Edn, eds G. G. Berntson, J. T. Cacioppo, and L. G. Tassinary (Cambridge: Cambridge University Press), 217-243.

Delgado, M. R., Nearing, K. I., LeDoux, J. E., and Phelps, E. A. (2008). Neural circuitry underlying the regulation of conditioned fear and its relation to extinction. Neuron 59, 829-838. doi: 10.1016/j.neuron.2008.06.029

Demaree, H. A., Schmeichel, B. J., Robinson, J. L., and Everhart, D. E. (2004). Behavioural, affective, and physiological effects of negative and positive emotional exaggeration. Cogn. Emot. 18, 1079-1097. doi: 10.1080/02699930441000085

Demaree, H. A., Schmeichel, B. J., Robinson, J. L., Pu, J., Everhart, D. E., and Berntson, G. G. (2006). Up- and down-regulating facial disgust: affective, vagal, sympathetic, and respiratory consequences. Biol. Psychol. 71, 90-99. doi: 10.1016/j.biopsycho.2005.02.006

Denson, T. F., Creswell, J. D., Terides, M. D., and Blundell, K. (2014). Cognitive reappraisal increases neuroendocrine reactivity to acute social stress and physical pain. Psychoneuroendocrinology 49, 69-78. doi: 10.1016/j.psyneuen.2014.07.003

Denson, T. F., Grisham, J. R., and Moulds, M. L. (2011). Cognitive reappraisal increases heart rate variability in response to an anger provocation. Motiv. Emot. 35, 14-22. doi: 10.1007/s11031-011-9201-5

Detenber, B. H., Simons, R. F., and Bennett Jr, G. G. (1998). Roll 'em!: the effects of picture motion on emotional responses. J. Broadcast. Electron. Media 42, 113-127. doi: 10.1080/08838159809364437

Deveney, C. M., and Pizzagalli, D. A. (2008). The cognitive consequences of emotion regulation: an ERP investigation. Psychophysiology 45, 435-444. doi: 10.1111/j.1469-8986.2007.00641.x

Di Simplicio, M., Costoloni, G., Western, D., Hanson, B., Taggart, P., and Harmer, C. J. (2012). Decreased heart rate variability during emotion regulation in subjects at risk for psychopathology. Psychol. Med. 42, 1775-1783. doi: 10.1017/S0033291711002479

Diers, K., Weber, F., Brocke, B., Strobel, A., and Schönfeld, S. (2014). Instructions matter: a comparison of baseline conditions for cognitive emotion regulation paradigms. Front. Psychol. 5:347. doi: 10.3389/fpsyg.2014.00347

Dillon, D. G., and LaBar, K. S. (2005). Startle modulation during conscious emotion regulation is arousal-dependent. Behav. Neurosci. 119, 1118-1124. doi: 10.1037/0735-7044.119.4.1118 
Driscoll, D., Tranel, D., and Anderson, S. W. (2009). The effects of voluntary regulation of positive and negative emotion on psychophysiological responsiveness. Int. J. Psychophysiol. 72, 61-66. doi: 10.1016/j.ijpsycho.2008.03.012

Edelmann, R. J., and Baker, S. R. (2002). Self-reported and actual physiological responses in social phobia. Br. J. Clin. Psychol. 41, 1-14. doi: 10.1348/014466502163732

Efinger, L., Thuillard, S., and Dan-Glauser, E. (2019). Distraction and reappraisal efficiency on immediate negative emotional responses: role of trait anxiety. Anxiety Stress Coping 32, 412-427. doi: 10.1080/10615806.2019.1597859

Egger, M., Davey Smith, G., Schneider, M., and Minder, C. (1997). Bias in metaanalysis detected by a simple, graphical test. Br. Med. J. 315, 629-634.

Eippert, F., Veit, R., Weiskopf, N., Erb, M., Birbaumer, N., and Anders, S. (2007). Regulation of emotional responses elicited by threat-related stimuli. Hum. Brain Mapp. 28, 409-423. doi: 10.1002/hbm.20291

Fechir, M., Schlereth, T., Purat, T., Kritzmann, S., Geber, C., Eberle, T., et al. (2008). Patterns of sympathetic responses induced by different stress tasks. Open Neurol. J. 2, 25-31. doi: 10.2174/1874205x008020 10025

Fitzpatrick, S., and Kuo, J. R. (2016). The impact of stimulus arousal level on emotion regulation effectiveness in borderline personality disorder. Psychiatry Res. 241, 242-248. doi: 10.1016/j.psychres.2016.05.004

Fuentes-Sánchez, N., Jaén, I., Escrig, M. A., Lucas, I., and Pastor, M. C. (2019). Cognitive reappraisal during unpleasant picture processing: subjective self-report and peripheral physiology. Psychophysiology 56:e13372. doi: 10.1111/psyp.13372

Giuliani, N. R., McRae, K., and Gross, J. J. (2008). The up- and down-regulation of amusement: experiential, behavioral, and autonomic consequences. Emotion 8, 714-719. doi: 10.1037/a0013236

Goldin, P. R., Moodie, C. A., and Gross, J. J. (2019). Acceptance versus reappraisal: behavioral, autonomic, and neural effects. Cogn. Affect. Behav. Neurosci. 19, 927-944. doi: 10.3758/s13415-019-00690-7

Golkar, A., Johansson, E., Kasahara, M., Osika, W., Perski, A., and Savic, I. (2014). The influence of work-related chronic stress on the regulation of emotion and on functional connectivity in the brain. PLOS ONE 9:e104550. doi: 10.1371/journal.pone.0104550

Gomez, P., Scholz, U., and Danuser, B. (2015). The down-regulation of disgust by implementation intentions: experiential and physiological concomitants. Appl. Psychophysiol. Biofeedback 40, 95-106. doi: 10.1007/s10484-015-9280-2

Greenwald, M. K., Cook, E. W., and Lang, P. J. (1989). Affective judgment and psychophysiological response: dimensional covariation in the evaluation of pictorial stimuli. J. Psychophysiol. 3, 51-64.

Grillon, C., and Baas, J. (2003). A review of the modulation of the startle reflex by affective states and its application in psychiatry. Clin. Neurophysiol. 114, 1557-1579. doi: 10.1016/s1388-2457(03)00202-5

Grillon, C., Quispe-Escudero, D., Mathur, A., and Ernst, M. (2015). Mental fatigue impairs emotion regulation. Emotion 15, 383-389. doi: 10.1037/emo0000058

Gross, J. J. (1998a). Antecedent-and response-focused emotion regulation: divergent consequences for experience, expression, and physiology. J. Pers. Soc. Psychol. 74, 224-237. doi: 10.1037/0022-3514.74.1.224

Gross, J. J. (1998b). The emerging field of emotion regulation: an integrative review. Rev. Gen. Psychol. 2, 271-299. doi: 10.1037/1089-2680.2. 3.271

Gross, J. J. (2002). Emotion regulation: affective, cognitive, and social consequences. Psychophysiology 39, 281-291. doi: 10.1017/s0048577201393198

Gross, J. J. (2015). Emotion regulation: Current status and future prospects. Psychol. Inq. 26, 1-26.

Gross, J. J., and Levenson, R. W. (1993). Emotional suppression: physiology, self-report, and expressive behavior. J. Pers. Soc. Psychol. 64, 970-986. doi: 10.1037//0022-3514.64.6.970

Gross, J. J., and Levenson, R. W. (1995). Emotion elicitation using films. Cogn. Emot. 9, 87-108. doi: 10.1080/02699939508408966

Gross, J. J., and Levenson, R. W. (1997). Hiding feelings: the acute effects of inhibiting negative and positive emotion. J. Abnorm. Psychol. 106, 95-103. doi: 10.1037/0021-843x.106.1.95

Gross, J. J., and Thompson, R. A. (2007). "Emotion regulation: conceptual foundations," in Handbook of Emotion Regulation, ed J. J. Gross (New York, NY: Guilford Press), 3-24
Gruber, J., Hay, A. C., and Gross, J. J. (2014). Rethinking emotion: cognitive reappraisal is an effective positive and negative emotion regulation strategy in bipolar disorder. Emotion 14, 388-396. doi: 10.1037/a00 35249

Hagemann, T., Levenson, R. W., and Gross, J. J. (2006). Expressive suppression during an acoustic startle. Psychophysiology 43, 104-112. doi: 10.1111/j.1469-8986.2006.00382.x

Hallam, G. P., Webb, T. L., Sheeran, P., Miles, E., Wilkinson, I. D., Hunter, M. D., et al. (2015). The neural correlates of emotion regulation by implementation intentions. PLoS ONE 10:e0119500. doi: 10.1371/journal.pone.01 19500

Hamm, A. O., Cuthbert, B. N., Globisch, J., and Vaitl, D. (1997). Fear and the startle reflex: blink modulation and autonomic response patterns in animal and mutilation fearful subjects. Psychophysiology 34, 97-107. doi: 10.1111/j.1469-8986.1997.tb02420.x

Hawk, L. W., and Cook, E. W. (2000). Independence of valence modulation and prepulse inhibition of startle. Psychophysiology 37, 5-12. doi: 10.1111/1469-8986.3710005

Hedges, L. V. (1982). Fitting categorical models to effect sizes from a series of experiments. J. Educ. Stat. 7, 119-137. doi: 10.3102/10769986007002119

Higgins, J. P., and Thompson, S. G. (2002). Quantifying heterogeneity in a meta-analysis. Stat. Med. 21, 1539-1558. doi: 10.1002/sim.1186

Hofmann, S. G., and Asmundson, G. J. (2008). Acceptance and mindfulness-based therapy: new wave or old hat? Clin. Psychol. Rev. 28, 1-16. doi: 10.1016/j.cpr.2007.09.003

Jackson, D. C., Malmstadt, J. R., Larson, C. L., and Davidson, R. J. (2000). Suppression and enhancement of emotional responses to unpleasant pictures. Psychophysiology 37, 515-522. doi: 10.1017/S0048577200990401

Jamieson, J. P., Mendes, W. B., and Nock, M. K. (2013). Improving acute stress responses: the power of reappraisal. Curr. Dir. Psychol. Sci. 22, 51-56. doi: 10.1177/0963721412461500

Jamieson, J. P., Nock, M. K., and Mendes, W. B. (2012). Mind over matter: reappraising arousal improves cardiovascular and cognitive responses to stress. J. Exp. Psychol. Gen. 141, 417-422. doi: 10.1037/a0025719

Joormann, J., and Vanderlind, W. M. (2014). Emotion regulation in depression the role of biased cognition and reduced cognitive control. Clin. Psychol. Sci. 2, 402-421. doi: 10.1177/2167702614536163

Kalisch, R. (2009). The functional neuroanatomy of reappraisal: time matters. Neurosci. Biobehav. Rev. 33, 1215-1226. doi: 10.1016/j.neubiorev.2009.06.003

Kalisch, R., Wiech, K., Critchley, H. D., Seymour, B., O'doherty, J. P., Oakley, D. A., et al. (2005). Anxiety reduction through detachment: subjective, physiological, and neural effects. J. Cogn. Neurosci. 17, 874-883. doi: 10.1162/0898929054021184

Kim, S. H., and Hamann, S. (2012). The effect of cognitive reappraisal on physiological reactivity and emotional memory. Int. J. Psychophysiol. 83, 348-356. doi: 10.1016/j.ijpsycho.2011.12.001

Kinner, V. L., Kuchinke, L., Dierolf, A. M., Merz, C. J., Otto, T., and Wolf, O. T. (2017). What our eyes tell us about feelings: tracking pupillary responses during emotion regulation processes. Psychophysiology 54, 508-518. doi: 10.1111/psyp.12816

Kirschbaum, C., Pirke, K.-M., and Hellhammer, D. H. (1993). The 'Trier Social Stress Test'-a tool for investigating psychobiological stress responses in a laboratory setting. Neuropsychobiology 28, 76-81. doi: 10.1159/000119004

Koole, S. L. (2009). The psychology of emotion regulation: an integrative review. Cogn. Emot. 23, 4-41. doi: 10.1080/02699930802619031

Kotwas, I., Micoulaud-Franchi, J. A., Khalfa, S., McGonigal, A., Bastien-Toniazzo, M., and Bartolomei, F. (2019). Subjective and physiological response to emotions in temporal lobe epilepsy and psychogenic non-epileptic seizures. J. Affect. Disord. 244, 46-53. doi: 10.1016/j.jad.2018.10.004

Kreibig, S. D. (2010). Autonomic nervous system activity in emotion: a review. Biol. Psychol. 84, 394-421. doi: 10.1016/j.biopsycho.2010.03.010

Kuhn, M., Wendt, J., Sjouwerman, R., Büchel, C., Hamm, A., and Lonsdorf, T. B. (2019). The neurofunctional basis of affective startle modulation in humans: evidence from combined facial electromyography and functional magnetic resonance imaging. Biol. Psychiatry 80, 548-558. doi: 10.1016/j.biopsych.2019.07.028

Kunzmann, U., Kupperbusch, C. S., and Levenson, R. W. (2005). Behavioral inhibition and amplification during emotional arousal: a comparison of 
two age groups. Psychol. Aging 20, 144-158. doi: 10.1037/0882-7974. 20.1.144

Lang, P. J. (1995). The emotion probe: studies of motivation and attention. Am. Psychol. 50, 372-385. doi: 10.1037/0003-066X.50.5.372

Lang, P. J., Bradley, M., and Cuthbert, B. (2009). International Affective Picture System (IAPS): Technical Manual and Affective Ratings. 1997. The Center for Research in Psychophysiology, University of Florida, Gainesville, FL.

Lapate, R. C., Rokers, B., Li, T., and Davidson, R. J. (2014). Nonconscious emotional activation colors first impressions: A regulatory role for conscious awareness. Psychol. Sci. 25, 349-357. doi: 10.1177/0956797613503175

Larsen, R. J. (2000). Toward a science of mood regulation. Psychol. Inq. 11, 129-141. doi: 10.1207/S15327965PLI1103_01

Lee, H., Shackman, A. J., Jackson, D. C., and Davidson, R. J. (2009). Test-retest reliability of voluntary emotion regulation. Psychophysiology 46, 874-879. doi: 10.1111/j.1469-8986.2009.00830.x

Leiberg, S., Eippert, F., Veit, R., and Anders, S. (2012). Intentional social distance regulation alters affective responses towards victims of violence: an FMRI study. Hum. Brain Mapp. 33, 2464-2476. doi: 10.1002/hbm.21376

Levenson, R. W., Ekman, P., and Friesen, W. V. (1990). Voluntary facial action generates emotion-specific autonomic nervous system activity. Psychophysiology 27, 363-384. doi: 10.1111/j.1469-8986.1990.tb02330.x

Levenson, R. W., and Gottman, J. M. (1983). Marital interaction: physiological linkage and affective exchange. J. Pers. Soc. Psychol. 45, 587-597. doi: 10.1037/0022-3514.45.3.587

Lissek, S., Orme, K., McDowell, D. J., Johnson, L. L., Luckenbaugh, D. A., Baas, J. M., et al. (2007). Emotion regulation and potentiated startle across affective picture and threat-of-shock paradigms. Biol. Psychol. 76, 124-133. doi: 10.1016/j.biopsycho.2007.07.002

Lohani, M., and Isaacowitz, D. M. (2014). Age differences in managing response to sadness elicitors using attentional deployment, positive reappraisal and suppression. Cogn. Emot. 28, 678-697 doi: 10.1080/02699931.2013.853648

Low, C. A., Stanton, A. L., and Bower, J. E. (2008). Effects of acceptance-oriented versus evaluative emotional processing on heart rate recovery and habituation. Emotion 8, 419-424. doi: 10.1037/1528-3542.8.3.419

Martins, B., Florjanczyk, J., Jackson, N. J., Gatz, M., and Mather, M. (2018). Age differences in emotion regulation effort: pupil response distinguishes reappraisal and distraction for older but not younger adults. Psychol. Aging 33, 338-349. doi: 10.1037/pag0000227

McRae, K., and Shiota, M. N. (2017). "Biological and physiological aspects of emotion regulation," in Emotion Regulation and Psychopathology in Children and Adolescents, eds C. A. Essau, S. Leblanc, and T. H. Ollendick (New York, NY: Oxford University Press), 43-59.

Morawetz, C., Bode, S., Baudewig, J., and Heekeren, H. R. (2017). Effective amygdala-prefrontal connectivity predicts individual differences in successful emotion regulation. Soc. Cogn. Affect. Neurosci. 12, 569-585. doi: 10.1093/scan/nsw169

Morawetz, C., Bode, S., Baudewig, J., Jacobs, A. M., and Heekeren, H. R. (2016a). Neural representation of emotion regulation goals. Hum. Brain Mapp. 37, 600-620. doi: 10.1002/hbm.23053

Morawetz, C., Kellermann, T., Kogler, L., Radke, S., Blechert, J., and Derntl, B. (2016b). Intrinsic functional connectivity underlying successful emotion regulation of angry faces. Soc. Cogn. Affect. Neurosci. 11, 1980-1991. doi: 10.1093/scan/nsw107

Obrist, P. A. (1981). Cardiovascular Psychophysiology. New York, NY: Springer Science \& Business Media.

Ochsner, K. N., Ray, R. D., Cooper, J. C., Robertson, E. R., Chopra, S., Gabrieli, J. D., et al. (2004). For better or for worse: neural systems supporting the cognitive down- and up-regulation of negative emotion. Neuroimage 23, 483-499. doi: 10.1016/j.neuroimage.2004.06.030

Ohira, H., Nomura, M., Ichikawa, N., Isowa, T., Iidaka, T., Sato, A., et al. (2006). Association of neural and physiological responses during voluntary emotion suppression. Neuroimage 29, 721-733. doi: 10.1016/j.neuroimage.2005.08.047

Öhman, A., and Soares, J. J. (1994). "Unconscious anxiety": phobic responses to masked stimuli. J. Abnormal Psychol. 103, 231-240. doi: 10.1037/0021-843X.103.2.231

Olsson, A., and Phelps, E. A. (2004). Learned fear of "unseen" faces after Pavlovian, observational, and instructed fear. Psychol. Sci. 15, 822-828. doi: 10.1111/j.0956-7976.2004.00762.x
Opitz, P. C., Lee, I. A., Gross, J. J., and Urry, H. L. (2014). Fluid cognitive ability is a resource for successful emotion regulation in older and younger adults. Front. Psychol. 5:609. doi: 10.3389/fpsyg.2014.00609

Ortner, C. N. M. (2015). Divergent effects of reappraisal and labeling internal affective feelings on subjective emotional experience. Motiv. Emot. 39, 563-570. doi: 10.1007/s11031-015-9473-2

Paret, C., Brenninkmeyer, J., Meyer, B., Yuen, K. S., Gartmann, N., Mechias, M.-L., et al. (2011). A test for the implementation-maintenance model of reappraisal. Front. Psychol. 2:216. doi: 10.3389/fpsyg.2011.00216

Peters, B. J., and Jamieson, J. P. (2016). The consequences of suppressing affective displays in romantic relationships: a challenge and threat perspective. Emotion 16, 1050-1066. doi: 10.1037/emo0000202

Peters, B. J., Overall, N. C., and Jamieson, J. P. (2014). Physiological and cognitive consequences of suppressing and expressing emotion in dyadic interactions. Int. J. Psychophysiol. 94, 100-107. doi: 10.1016/j.ijpsycho.2014.07.015

Piper, M. E., and Curtin, J. J. (2006). Tobacco withdrawal and negative affect: An analysis of initial emotional response intensity and voluntary emotion regulation. J. Abnorm. Psychol. 115, 96-102. doi: 10.1037/0021-843X.115.1.96

Plieger, T., Melchers, M., Vetterlein, A., Görtz, J., Kuhn, S., Ruppel, M., et al. (2017). The serotonin transporter polymorphism (5-HTTLPR) and coping strategies influence successful emotion regulation in an acute stress situation: physiological evidence. Int. J. Psychophysiol. 114, 31-37. doi: 10.1016/j.ijpsycho.2017.02.006

Reinecke, A., Filippini, N., Berna, C., Western, D. G., Hanson, B., Cooper, M. J., et al. (2015). Effective emotion regulation strategies improve fMRI and ECG markers of psychopathology in panic disorder: implications for psychological treatment action. Transl. Psychiatry 5:e673. doi: 10.1038/tp.2015.160

Richards, J. M., and Gross, J. J. (1999). Composure at any cost? The cognitive consequences of emotion suppression. Pers. Soc. Psychol. Bull. 25, 1033-1044. doi: 10.1177/01461672992511010

Roberts, N. A., Levenson, R. W., and Gross, J. J. (2008). Cardiovascular costs of emotion suppression cross ethnic lines. International Journal of Psychophysiology, 70, 82-87. doi: 10.1016/j.ijpsycho.2008.06.003

Robinson, J. L., and Demaree, H. A. (2009). Experiencing and regulating sadness: physiological and cognitive effects. Brain Cogn. 70, 13-20. doi: 10.1016/j.bandc.2008.06.007

Rohrmann, S., Hopp, H., Schienle, A., and Hodapp, V. (2009). Emotion regulation, disgust sensitivity, and psychophysiological responses to a disgust-inducing film. Anxiety Stress Coping 22, 215-236. doi: 10.1080/10615800802016591

Rosen, J. B., and Davis, M. (1988). Enhancement of acoustic startle by electrical stimulation of the amygdala. Behav. Neurosci. 102, 195-202. doi: 10.1037/0735-7044.102.2.195

Rosenthal, R., and Rubin, D. B. (1982). Comparing effect sizes of independent studies. Psychol. Bull. 92, 500-504. doi: 10.1037/0033-2909.92.2.500

Roth, G., Benita, M., Amrani, C., Shachar, B. H., Asoulin, H., Moed, A., et al. (2014). Integration of negative emotional experience versus suppression: addressing the question of adaptive functioning. Emotion 14, 908-919. doi: 10.1037/a0037051

Schaefer, S. M., Jackson, D. C., Davidson, R. J., Aguirre, G. K., Kimberg, D. Y., and Thompson-Schill, S. L. (2002). Modulation of amygdalar activity by the conscious regulation of negative emotion. J. Cogn. Neurosci. 14, 913-921. doi: 10.1162/089892902760191135

Schmahl, C., Herpertz, S. C., Bertsch, K., Ende, G., Flor, H., Kirsch, P., et al. (2014). Mechanisms of disturbed emotion processing and social interaction in borderline personality disorder: state of knowledge and research agenda of the German Clinical Research Unit. Borderline Pers. Disord. Emot. Dysregul. 1:12. doi: 10.1186/2051-6673-1-12

Schupp, H. T., Cuthbert, B. N., Bradley, M. M., Birbaumer, N., and Lang, P. J. (1997). Probe P3 and blinks: two measures of affective startle modulation. Psychophysiology 34, 1-6. doi: 10.1111/j.1469-8986.1997.tb0 2409.x

Sheppes, G., Catran, E., and Meiran, N. (2009). Reappraisal (but not distraction) is going to make you sweat: physiological evidence for self-control effort. Int. J. Psychophysiol. 71, 91-96. doi: 10.1016/j.ijpsycho.2008.06.006

Shermohammed, M., Mehta, P. H., Zhang, J., Brandes, C. M., Chang, L. J., and Somerville, L. H. (2017). Does psychosocial stress impact cognitive reappraisal? Behavioral and neural evidence. J. Cogn. Neurosci. 29, 1803-1816. doi: 10.1162/jocn_a_01157 
Shiota, M. N., and Levenson, R. W. (2009). Effects of aging on experimentally instructed detached reappraisal, positive reappraisal, and emotional behavior suppression. Psychol. Aging 24, 890-900. doi: 10.1037/a00 17896

Shiota, M. N., and Levenson, R. W. (2012). Turn down the volume or change the channel? Emotional effects of detached versus positive reappraisal. J. Pers. Soc. Psychol. 103, 416-429. doi: 10.1037/a0029208

Shiota, M. N., Neufeld, S. L., Yeung, W. H., Moser, S. E., and Perea, E. F. (2011). Feeling good: autonomic nervous system responding in five positive emotions. Emotion 11, 1368-1378. doi: 10.1037/a0024278 .

Siegel, E. H., Sands, M. K., Van den Noortgate, W., Condon, P., Chang, Y., Dy, J., et al. (2018). Emotion fingerprints or emotion populations? A meta-analytic investigation of autonomic features of emotion categories. Psychol. Bull. 144, 343-393. doi: 10.1037/bul0000128

Simons, R. F., Detenber, B. H., Roedema, T. M., and Reiss, J. E. (1999). Emotion processing in three systems: the medium and the message. Psychophysiology 36, 619-627. doi: 10.1111/1469-8986.3650619

Soto, J. A., Lee, E. A., and Roberts, N. A. (2016). Convergence in feeling, divergence in physiology: how culture influences the consequences of disgust suppression and amplification among European Americans and Asian Americans. Psychophysiology 53, 41-51. doi: 10.1111/psy p.12579

Stemmler, G. (2004). "Physiological processes during emotion," in The Regulation of Emotion, eds P. Philippot, R. S. Feldman, P. Philippot, and R. S. Feldman (Mahwah, NJ: Lawrence Erlbaum Associates Publishers). 33-70.

Stiller, A.-K., Kattner, M. F., Gunzenhauser, C., and Schmitz, B. (2019). The effect of positive reappraisal on the availability of selfcontrol resources and self-regulated learning. Educ. Psychol. 39, 86-111. doi: 10.1080/01443410.2018.1524851

Strauss, G. P., Ossenfort, K. L., and Whearty, K. M. (2016). Reappraisal and distraction emotion regulation strategies are associated with distinct patterns of visual attention and differing levels of cognitive demand. PLOS ONE 11:e0162290. doi: 10.1371/journal.pone.0162290

Svaldi, J., Caffier, D., and Tuschen-Caffier, B. (2010). Emotion suppression but not reappraisal increases desire to binge in women with binge eating disorder. Psychother. Psychosom. 79, 188-190. doi: 10.1159/000296138

Tassinary, L. G., Cacioppo, J. T., and Vanman, E. J. (2007). “The skeletomotor system: surface electromyography," in Handbook of Psychophysiology, eds J. T. Cacioppo, L. G. Tassinary, and G. G. Berntson (New York, NY: Cambridge University Press), 267-299.

Thompson, S. G., and Sharp, S. J. (1999). Explaining heterogeneity in metaanalysis: a comparison of methods. Stat. Med. 18, 2693-2708. doi: 10.1002/ (SICI)1097-0258(19991030)18:20<2693::AID-SIM235>3.0.CO;2-V

Urry, H. L. (2009). Using reappraisal to regulate unpleasant emotional episodes: goals and timing matter. Emotion 9, 782-797. doi: 10.1037/a00 17109

Urry, H. L. (2010). Seeing, thinking, and feeling: emotion-regulating effects of gaze-directed cognitive reappraisal. Emotion 10, 125-135. doi: 10.1037/a0017434

Urry, H. L., van Reekum, C. M., Johnstone, T., and Davidson, R. J. (2009). Individual differences in some (but not all) medial prefrontal regions reflect cognitive demand while regulating unpleasant emotion. Neuroimage 47, 852-863. doi: 10.1016/j.neuroimage.2009.05.069
Urry, H. L., van Reekum, C. M., Johnstone, T., Kalin, N. H., Thurow, M. E., Schaefer, H. S., et al. (2006). Amygdala and ventromedial prefrontal cortex are inversely coupled during regulation of negative affect and predict the diurnal pattern of cortisol secretion among older adults. J. Neurosci. 26, 4415-4425. doi: 10.1523/jneurosci.3215-05.2006

Uy, C. C., Jeffery, I. A., Wilson, M., Aluru, V., Madan, A., Lu, Y., et al. (2013). Autonomic mechanisms of emotional reactivity and regulation. Sci. Res. 43, 669-675. doi: 10.4236/psych.2013.48095

van Reekum, C. M., Johnstone, T., Urry, H. L., Thurow, M. E., Schaefer, H. S. Alexander, A. L., et al. (2007). Gaze fixations predict brain activation during the voluntary regulation of picture-induced negative affect. Neuroimage 36, 1041-1055. doi: 10.1016/j.neuroimage.2007.03.052

VanOyen Witvliet, C., and Vrana, S. R. (1995). Psychophysiological responses as indices of affective dimensions. Psychophysiology 32, 436-443. doi: 10.1111/j.1469-8986.1995.tb02094.x

Vrana, S. R., Spence, E. L., and Lang, P. J. (1988). The startle probe response: a new measure of emotion? J. Abnorm. Psychol. 97, 487-491. doi: 10.1037/0021-843X.97.4.487

Wangelin, B. C., Bradley, M. M., Kastner, A., and Lang, P. J. (2012). Affective engagement for facial expressions and emotional scenes: the influence of social anxiety. Biol. Psychol. 91, 103-110. doi: 10.1016/j.biopsycho.2012.05.002

Webb, T. L., Miles, E., and Sheeran, P. (2012). Dealing with feeling: a meta-analysis of the effectiveness of strategies derived from the process model of emotion regulation. Psychol. Bull. 138, 775-808. doi: 10.1037/a00 27600

Williams, L. E., Bargh, J. A., Nocera, C. C., and Gray, J. R. (2009). The unconscious regulation of emotion: nonconscious reappraisal goals modulate emotional reactivity. Emotion 9, 847-854. doi: 10.1037/a0017745

Wolgast, M., Lundh, L.-G., and Viborg, G. (2011). Cognitive reappraisal and acceptance: an experimental comparison of two emotion regulation strategies. Behav. Res. Ther. 49, 858-866. doi: 10.1016/j.brat.2011.09.011

Wu, M., Liang, Y., Wang, Q., Zhao, Y., and Zhou, R. (2016). Emotion dysregulation of women with premenstrual syndrome. Sci. Rep. 6:38501. doi: 10.1038/srep38501

Yuan, J., Liu, Y., Ding, N., and Yang, J. (2014). The regulation of induced depression during a frustrating situation: benefits of expressive suppression in Chinese individuals. PLoS ONE 9:e97420. doi: 10.1371/journal.pone.0097420

Zaehringer, J., Schmahl, C., Ende, G., and Paret, C. (2018). Emotionmodulated startle reflex during reappraisal: probe timing and behavioral correlates. Behav. Neurosci. 132, 573-579. doi: 10.1037/bne00 00271

Conflict of Interest: The authors declare that the research was conducted in the absence of any commercial or financial relationships that could be construed as a potential conflict of interest.

Copyright (C) 2020 Zaehringer, Jennen-Steinmetz, Schmahl, Ende and Paret. This is an open-access article distributed under the terms of the Creative Commons Attribution License (CC BY). The use, distribution or reproduction in other forums is permitted, provided the original author(s) and the copyright owner(s) are credited and that the original publication in this journal is cited, in accordance with accepted academic practice. No use, distribution or reproduction is permitted which does not comply with these terms. 